\title{
SRB Measures as Zero-noise Limits
}

\author{
William Cowieson ${ }^{1}$ and Lai-Sang Young ${ }^{2}$
}

\begin{abstract}
We consider zero-noise limits of random perturbations of dynamical systems and examine, in terms of the continuity of entropy and Lyapunov exponents, circumstances under which these limits are SRB measures. The ideas of this general discussion are then applied to specific classes of attractors. We prove, for example, that partially hyperbolic attractors with one-dimensional center subbundles always admit SRB measures.
\end{abstract}

In this paper we attempt to address the following question:

To a deterministic dynamical system we add small random perturbations and consider the one-parameter family of Markov chains parametrized by $\varepsilon$ where $\varepsilon>0$ is the noise level. What can be said about their limiting distributions as $\varepsilon$ tends to zero?

More precisely, consider a dynamical system generated by a map $f: M \rightarrow M$, and let $\mu_{\varepsilon}$ denote the probability measure on $M$ that is invariant under the Markov chain corresponding to noise level $\varepsilon$. As $\varepsilon \rightarrow 0$, the weak limits of $\mu_{\varepsilon}$, which we call zero-noise limits, are special invariant measures of $f$. The importance of this class of invariant measures is clear: assuming that the real world is perpetually slightly noisy, they represent idealizations of what we see. These ideas go back to Kolmogorov; see [S2].

Atomic measures concentrated at attractive fixed points or periodic orbits of $f$ are obvious examples of zero-noise limits. We are primarily interested in the "chaotic" case, where the zero-noise limit has a positive Lyapunov exponent. There are reasons to believe that under fairly general conditions, SRB measures may be natural candidates for zero-noise limits. One of these reasons will be explained below. Indeed, in some situations, this is a viable way of proving the existence of SRB measures.

Among invariant measures preserved by diffeomorphisms, SRB measures are characterized by the fact that they have a positive Lyapunov exponent and satisfy the entropy equality

$$
h_{\mu}=\lambda_{\mu}^{+}
$$

where $h_{\mu}$ denotes the metric entropy of $f$ with respect to the measure $\mu$ and $\lambda_{\mu}^{+}$denotes the integral of the sum of the positive Lyapunov exponents counted with multiplicity $([\mathrm{P}]$, [LS], [L], [LY1]).

Turning to the noisy situation, a large class of random perturbations can be represented by random maps. In this setting, a similar equality, namely

$$
h_{\mu_{\varepsilon}}=\lambda_{\mu_{\varepsilon}}^{+},
$$

\footnotetext{
${ }^{1}$ Courant Inst. of Math. Sc., 251 Mercer St., New York, NY 10012; email cowieson@cims.nyu.edu

${ }^{2}$ Courant Inst. of Math. Sc., 251 Mercer St., New York, NY 10012; email lsy@cims.nyu.edu. This research is partially supported by a grant from the NSF
} 
holds for every invariant measure $\mu_{\varepsilon}$ of the Markov chain provided that the transition probabilities have densities with respect to Lebesgue measure [LY2].

Comparing (1) and (2), we see that if (2) can be passed to the limit $\varepsilon=0$, then any limit point of $\mu_{\varepsilon}$ having a positive Lyapunov exponent is an SRB measure. This last supposition, however, is not always valid, for both entropy and Lyapunov exponents are known to have discontinuities.

The purpose of this paper is to bring to the foreground some facts regarding the limiting behavior of entropy and Lyapunov exponents as $\varepsilon \rightarrow 0$, and to identify some simple situations in which the passage of (2) to this limit is guaranteed. A consequence of this line of reasoning is the existence of SRB measures for certain classes of maps, an example of which is the following:

Theorem C. Partially hyperbolic systems with one-dimensional center subbundles have SRB measures.

The method used in the proof of Theorem C, namely by adding noise to a dynamical system and obtaining an SRB measure as a zero-noise limit, is likely to be viable in other situations.

The main results of this paper (Theorems A, B, and C) are stated in Section 2 after a review of some definitions and facts. Section 3 contains a general discussion of the basic issues regarding the continuity of entropy and Lyapunov exponents. The aim of this discussion is to pinpoint exactly what needs to be controlled. The proofs of Theorems A, B and $\mathrm{C}$ are carried out in Sections 4 and 5.

Throughout this paper, $M$ is a compact Riemannian manifold with normalized volume $m$, and $f: M \rightarrow M$ is a differentiable map.

\section{Background Information}

This section contains a brief review of the definitions and elementary facts used in the formulation of our main results.

\section{$1.1 \quad$ SRB measures}

We assume here that $f$ is a $C^{2}$ diffeomorphism.

Definition 1. An $f$-invariant Borel probability measure $\mu$ on $M$ is called a Sinai-RuelleBowen measure or SRB measure if $f$ has a positive Lyapunov exponent $\mu$-a.e. and the conditional measures of $\mu$ on unstable manifolds are absolutely continuous with respect to the Riemannian measures on these leaves.

This terminology was first introduced in [ER]. For more detail, see [Y2] and the references therein. Very briefly, the idea is as follows: For dissipative, chaotic dynamical systems, such as those with attractors, SRB measures are as close to volume as invariant measures can be in that they have densities in unstable directions. 
An important property of ergodic SRB measures with no zero Lyapunov exponents is that they are physical; i.e., there is a positive Lebesgue measure set $V \subset M$ such that for all continuous functions $\varphi$ on $M, \frac{1}{n} \sum_{i=0}^{n-1} \varphi\left(f^{i}(x)\right) \rightarrow \int \varphi d \mu$ as $n \rightarrow \infty$ for every $x \in V$.

Let $\mu$ be an $f$-invariant Borel probability measure. We let $h_{\mu}$ denote the metric entropy of $f$ with respect to $\mu$, and let $\lambda_{1}(x)>\cdots>\lambda_{r(x)}(x)$ denote the distinct Lyapunov exponents of $f$ at $x$, the multiplicity of $\lambda_{i}$ being $m_{i}$. By Oseledec's theorem, we know that Lyapunov exponents are defined $\mu$-a.e. We write $a^{+}=\max \{a, 0\}$, and let

$$
\lambda_{\mu}^{+}:=\int \sum_{i=1}^{r(x)} \lambda_{i}^{+}(x) m_{i}(x) d \mu .
$$

Theorem 1. (i) [P], [LS] Let $\mu$ be an SRB measure. Then

$$
h_{\mu}=\lambda_{\mu}^{+} \text {. }
$$

(ii) [L], [LY1] Conversely, if the formula in (i) holds and $h_{\mu}>0$, then $\mu$ is an $S R B$ measure.

Remark. Let $\mu=\int \mu_{e} d \pi$ be the ergodic decomposition of $\mu$. If the entropy formula in (i) holds for $\mu$, then it holds also for $\pi$-a.e. $\mu_{e}$. (See e.g. [LY1].)

Part (ii) of Theorem 1 together with the remark above is our main tool for identifying certain invariant measures as SRB measures.

\subsection{Random perturbations}

Let $\mathcal{M}$ be the set of Borel probability measures on $M$ endowed with the weak topology. We consider a family of transition probabilities $\mathcal{X}=\{p(\cdot \mid x)\}$ on $M$; i.e., associated with each $x \in M$ is a probability $p(\cdot \mid x) \in \mathcal{M}$. By a hopefully benign abuse of language, we will refer to $\mathcal{X}$ as a Markov chain even though no initial distribution is specified. We say $\mu \in \mathcal{M}$ is an invariant or stationary measure for $\mathcal{X}$ if for every continuous function $\varphi$ on $M$,

$$
\int \varphi d(\mathcal{L} \mu):=\iint \varphi(y) d p(y \mid x) d \mu(x)=\int \varphi d \mu .
$$

Here $\mathcal{L}: \mathcal{M} \rightarrow \mathcal{M}$ is the transfer operator associated with $\mathcal{X}$, and stationary measures are precisely the fixed points of $\mathcal{L}$. We consider only Markov chains for which $\mathcal{L}$ is continuous. This together with the compactness of $M$ ensures that $\mathcal{X}$ has at least one stationary measure. A stationary measure $\mu$ is ergodic if the only fixed points of $\mathcal{L}^{*}: L^{1}(\mu) \rightarrow L^{1}(\mu)$ are the constant functions.

We are primarily interested in Markov chains which are perturbations of dynamical systems. Let $\delta_{x}$ denote the Dirac measure at $x$.

Definition 2. A small random perturbation of $f: M \rightarrow M$ is a one-parameter family of Markov chains $\mathcal{X}^{\varepsilon}, \varepsilon>0$, given by transition probabilities $\left\{p^{\varepsilon}(\cdot \mid x): x \in M\right\}$ which satisfy $p^{\varepsilon}(\cdot \mid x) \rightarrow \delta_{f(x)}$ uniformly in $x$ as $\varepsilon \rightarrow 0$. We also allow $\varepsilon$ to be a discrete parameter, i.e., a sequence of numbers converging to 0 . 
Definition 3. We say $\mu \in \mathcal{M}$ is a zero-noise limit of a small random perturbation $\mathcal{X}^{\varepsilon}$ of $f$ if, in the weak topology of $\mathcal{M}, \mu$ is a limit point of $\mu_{\varepsilon}$ as $\varepsilon \rightarrow 0$ where $\mu_{\varepsilon}$ are stationary measures of $\mathcal{X}^{\varepsilon}$.

The compactness of $\mathcal{M}$ ensures that every $\mathcal{X}^{\varepsilon}$ has a zero-noise limit. This limit, in general, need not be unique. The following is a related notion:

Definition 4. An $f$-invariant measure $\mu$ is said to be stochastically stable with respect to small random perturbations from a class of Markov chains $\mathcal{F}$ if for every small random perturbation $\mathcal{X}^{\varepsilon}$ of $f$ in $\mathcal{F}$ and every collection of stationary measures $\mu_{\varepsilon}, \mu_{\varepsilon} \rightarrow \mu$ weakly as $\varepsilon \rightarrow 0$.

Stochastic stability has been studied by many authors; see e.g. [K3]. In the present paper, we do not begin with an invariant measure $\mu$ of $f$ and ask if it is stable. Instead, we begin with a small random perturbation of $f$ and use it to identify some invariant measures, namely the zero-noise limits of the perturbation.

Let " $\mu<<\nu$ " be the notation for " $\mu$ is absolutely continuous with respect to $\nu$ ". The following is an elementary exercise.

Lemma 1. (1) If $p(\cdot \mid x)<<m$ for every $x$, then every stationary measure is $<<m$.

(2) Zero-noise limits are $f$-invariant measures.

\subsection{Random maps}

We now turn to Markov chains on $M$ defined by compositions of i.i.d. random diffeomorphisms. Let $\Omega=\operatorname{Diff}^{r}(M), 1 \leq r \leq \infty$, denote the space of $C^{r}$ diffeomorphisms of $M$ with the $C^{r}$ topology. Given a Borel probability measure $\nu$ on $\Omega$, we define a collection $\mathcal{X}$ of transition probabilities on $M$ by

$$
p(A \mid x)=\nu(\{g: g(x) \in A\}) .
$$

The associated transfer operator is given by $\mathcal{L} \mu=\int_{\Omega} g_{*} \mu d \nu(g)$ where $g_{*} \mu=\mu \circ g^{-1}$.

Markov chains of this type have representations as skew products. More precisely, let $\theta: \Omega^{\mathbf{N}} \rightarrow \Omega^{\mathbf{N}}$ be the shift operator and define a map $F^{+}: \Omega^{\mathbf{N}} \times M \rightarrow \Omega^{\mathbf{N}} \times M$ by

$$
F^{+}(\omega, x)=\left(\theta \omega, \omega_{1}(x)\right)
$$

where $\omega=\left(\omega_{1}, \omega_{2}, \ldots\right)$ is an element of $\Omega^{\mathbf{N}}$.

Lemma 2. Let $\mathcal{X}, \nu$, and $F^{+}$be as above, and let $\mu$ be a probability on $M$. Then $\mu$ is stationary/ergodic for $\mathcal{X}$ if and only if $\nu^{\mathbf{N}} \times \mu$ is invariant/ergodic for $F^{+}$.

This representation makes it possible to define Lyapunov exponents and entropy for random maps. For each $\omega=\left(\omega_{1}, \omega_{2}, \ldots\right)$, it is a great convenience to write

$$
\omega^{n}:=\omega_{n} \circ \ldots \circ \omega_{1} .
$$

That is, we identify the probability space with the space of maps and free ourselves of the usual, somewhat cumbersome notation $f_{\omega}^{n}=f_{n} \circ \ldots \circ f_{1}$, where $f_{i}=f_{i}(\omega)$. 
Provided that

$$
\int \log ^{+}\left\|D \omega_{1}(x)\right\| d\left(\mu \times \nu^{\mathbf{N}}\right)<\infty
$$

where $\log ^{+} a=\max \{\log a, 0\}$, Oseledec's theorem $([\mathrm{O}])$ guarantees that for $\left(\mu \times \nu^{\mathbf{N}}\right)$-a.e. $(x, \omega)$, there exist numbers $r(x) \in \mathbf{N}, \lambda_{1}(x)>\ldots>\lambda_{r}(x) \geq-\infty$, and a filtration $T_{x} M=$ $V_{1}(x, \omega) \supset \ldots V_{r}(x, \omega) \supset V_{r+1}=\{0\}$, such that the limit

$$
\lambda(x, v, \omega):=\lim _{n \rightarrow \infty} \frac{1}{n} \log \left\|D \omega^{n}(x) \cdot v\right\|
$$

exists and is equal to $\lambda_{i}(x)$ for all $v \in V_{i}(x, \omega) \backslash V_{i+1}(x, \omega)$. The numbers $\lambda_{i}(x)$ together with their multiplicities $m_{i}(x):=\operatorname{dim} V_{i}(x, \omega)-\operatorname{dim} V_{i+1}(x, \omega)$ (which depend only on $x$ ) are called the Lyapunov exponents of the random system. If $\left(F^{+}, \mu \times \nu^{\mathbf{N}}\right)$ is ergodic, then $r, \lambda_{i}$ and $m_{i}$ are constant $\mu$-a.e. The quantity that will appear in our theorems is

$$
\lambda_{\mu}^{+}:=\int \sum_{i=1}^{r(x)} \lambda_{i}^{+}(x) m_{i}(x) d \mu .
$$

Finally, we define a "pathwise" notion of entropy for random maps. Let $\alpha=\left\{A_{1}, \ldots, A_{n}\right\}$ be a finite partition of $M$. As usual, $H_{\mu}(\alpha)=-\sum \mu\left(A_{i}\right) \log \mu\left(A_{i}\right)$,

$$
H_{\mu}(\omega, \alpha, n)=H_{\mu}\left(\bigvee_{i=0}^{n-1}\left(\omega^{i}\right)^{-1} \alpha\right), \quad H_{\mu}(\alpha, n)=\int H_{\mu}(\omega, \alpha, n) d \nu^{\mathbf{N}}(\omega)
$$

and

$$
h_{\mu}(\alpha):=\lim _{n \rightarrow \infty} \frac{1}{n} H_{\mu}(\alpha, n) .
$$

This limit exists and is equal to the infimum over $n$. A random version of the Shannon-

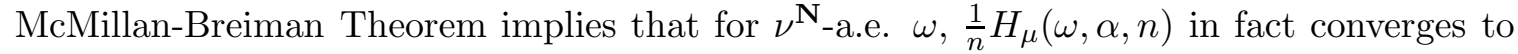
$h_{\mu}(\alpha)$ as $n \rightarrow \infty$. The entropy of the random map process is defined to be

$$
h_{\mu}=\sup _{\alpha} h_{\mu}(\alpha)
$$

For more detail, see $[\mathrm{K} 2]$ and $[\mathrm{LQ}]$.

\subsection{Local entropy}

The following notion was introduced for a single map by Bowen [Bo]. We give the version for random maps. Let $\omega=\left(\omega_{1}, \omega_{2}, \ldots\right)$ be a sequence of maps of a space $X$ to itself. For $\rho>0$ and $x \in X$, let

$$
B^{+}(x, \omega, \rho)=\left\{y \in X: d\left(\omega^{j}(x), \omega^{j}(y)\right)<\rho, j=0,1,2, \ldots\right\} .
$$

For $\delta>0$ and a subset $K \subset X$ define $r(\delta, n, K)$ to be the minimal cardinality of a $(\delta, n)$ spanning set of $K$. (A set $E$ is a $(\delta, n)$-spanning set of $K$ if for every $y \in K$ there is an 
$x \in E$ such that $d\left(\omega^{k}(x), \omega^{k}(y)\right) \leq \delta$ for all $0 \leq k<n$.) The $\rho$-local entropy of the sequence $\omega$ is

$$
h_{l o c}(\omega, \rho):=\sup _{x \in X} \lim _{\delta \rightarrow 0} \limsup _{n \rightarrow \infty} \frac{1}{n} \log r\left(\delta, n, B^{+}(x, \omega, \rho)\right)
$$

and for a random map process with law $\nu$, we define

$$
h_{\nu, l o c}(\rho)=\int h_{l o c}(\omega, \rho) d \nu^{\mathbf{N}} .
$$

Lemma 3. Let $\mu$ be a stationary measure for a random map process on $M$ with law $\nu$. Then for any finite Borel partition $\alpha$ of $M$ with $\operatorname{diam}(\alpha) \leq \rho$,

$$
h_{\mu} \leq h_{\mu}(\alpha)+h_{\nu, l o c}(\rho) .
$$

This is the randomized version of Theorem 3.5 in [Bo]. We omit the proof, which is carried out one sequence at a time and follows closely that for a single map. Note that this proof uses the finite dimensionality of the phase space.

\section{Statements of Results}

Let $\Omega=\operatorname{Diff}^{r}(M)$ for some $1 \leq r \leq \infty$. For $r<\infty$, the usual $C^{r}$-norm defines a metric on $\Omega$. For $r=\infty$, we use the metric $\bar{d}_{\infty}(f, g)=\sum_{s=0}^{\infty} \frac{1}{2^{s}} \min \left(1,\|f-g\|_{C^{s}}\right)$.

Standing Hypotheses In the rest of this paper, $\mathcal{X}^{\varepsilon}$ is assumed to be a Markov chain on $M$ which satisfies

(H1) $p^{\varepsilon}(\cdot \mid x)<<m$ for every $x$,

(H2) $\mathcal{X}^{\varepsilon}$ is defined by i.i.d. random maps with law $\nu^{\varepsilon}, \nu^{\varepsilon}$ being a probability on $\Omega=$ $\operatorname{Diff}^{r}(M)$ for some $r$.

The following shorthand will be used: " $\left(\mathcal{X}^{\varepsilon}, \mu_{\varepsilon}\right) \rightarrow\left(f, \mu_{0}\right)$ " means that $\mathcal{X}^{\varepsilon}$ is a small random perturbation of $f$ satisfying (H1) and (H2), $\mu_{\varepsilon}$ is a stationary measure of $\mathcal{X}^{\varepsilon}$ (it may or may not be unique), and $\mu_{\varepsilon} \rightarrow \mu_{0}$ weakly as $\varepsilon \rightarrow 0$. The support of $\nu^{\varepsilon}$ is denoted by $\operatorname{supp}\left(\nu^{\varepsilon}\right)$. By Lemma 1 , if $\left(\mathcal{X}^{\varepsilon}, \mu_{\varepsilon}\right) \rightarrow\left(f, \mu_{0}\right)$, then $\mu_{\varepsilon}<<m$ for every $\varepsilon>0$ and $\mu_{0}$ is $f$-invariant.

We remark that given $f$, it is easy to construct small random perturbations with properties (H1) and (H2). To demonstrate, let $f$ be $C^{r}$ and define $\mathcal{X}^{\varepsilon}$ by random maps of the form $g_{\mathbf{t}}=\varphi_{t_{k}}^{k} \circ \ldots \circ \varphi_{t_{1}}^{1} \circ f$, where $\varphi_{t}^{i}$ is the time-t-map of the flow generated by a $C^{r}$ vector field $X^{i}$, and $\mathbf{t}=\left(t_{1}, \ldots, t_{k}\right)$ is chosen at random from $[-\varepsilon, \varepsilon]^{k}$ with uniform distribution. (H1) is satisfied if $X^{1}, \cdots, X^{k}$ span the tangent space at each point, and (H2) is satisfied whenever $\mathcal{N}$ is a $C^{r}$ neighborhood of $f$ and $\varepsilon$ is small enough. We choose a stationary $\mu_{\varepsilon}$ for each $\mathcal{X}^{\varepsilon}$ and, if $\lim _{\varepsilon \rightarrow 0} \mu_{\varepsilon}$ exists, then we call the limit $\mu_{0}$ and write $\left(\mathcal{X}^{\varepsilon}, \mu_{\varepsilon}\right) \rightarrow\left(f, \mu_{0}\right)$. If $\lim _{\varepsilon \rightarrow 0} \mu_{\varepsilon}$ does not exist, then we pass to a convergent subsequence $\mu_{\varepsilon_{n}} \rightarrow \mu_{0}$ and write $\left(\mathcal{X}_{\varepsilon_{n}}, \mu_{\varepsilon_{n}}\right) \rightarrow\left(f, \mu_{0}\right)$. Either way, $\mu_{0}$ is a zero-noise limit of stationary measures $\mu_{\varepsilon}<<m$. 


\section{$2.1 \quad$ A result for $C^{\infty}$ maps}

For this result $\Omega=\operatorname{Diff}^{\infty}(M)$.

Theorem A. Let $f \in \Omega$, and consider $\mathcal{X}^{\varepsilon}$ with the property that for all $\varepsilon>0$, $\operatorname{supp}\left(\nu^{\varepsilon}\right) \subset \mathcal{N}$ where $\mathcal{N} \subset \Omega$ is bounded. If $\left(\mathcal{X}^{\varepsilon}, \mu_{\varepsilon}\right) \rightarrow\left(f, \mu_{0}\right)$, then

$$
\limsup _{\varepsilon \rightarrow 0} h_{\mu_{\varepsilon}} \leq h_{\mu_{0}}
$$

Thus,

$$
\text { if } \quad \limsup _{\varepsilon \rightarrow 0} \lambda_{\mu_{\varepsilon}}^{+} \geq \lambda_{\mu_{0}}^{+}, \text {then } h_{\mu_{0}}=\lambda_{\mu_{0}}^{+},
$$

and it follows that almost every ergodic component of $\mu_{0}$ with a positive Lyapunov exponent is an SRB measure.

Remarks. (1) Theorem A is in fact valid for random perturbations that are not necessarily supported on a bounded subset of $\Omega$, provided there is suitable control of the tail part of the distribution. We leave this generalization to the reader. (See $[\mathrm{KY}]$.)

(2) We will see in Section 3.1 that the condition in equation (3) above is equivalent to the continuity of the sum of positive Lyapunov exponents as $\varepsilon \rightarrow 0$. When this continuity holds, Theorem A says that zero-noise limits have only two kinds of ergodic components: SRB measures and measures with no expansion.

(3) This continuity assumption, however, is not always fulfilled, as is evident in the "figure-eight attractor" depicted below. This attractor supports a unique invariant probability measure, which perforce is the zero-noise limit of any perturbation. Being a Dirac measure at the saddle fixed point, it is clearly not of the type described above.

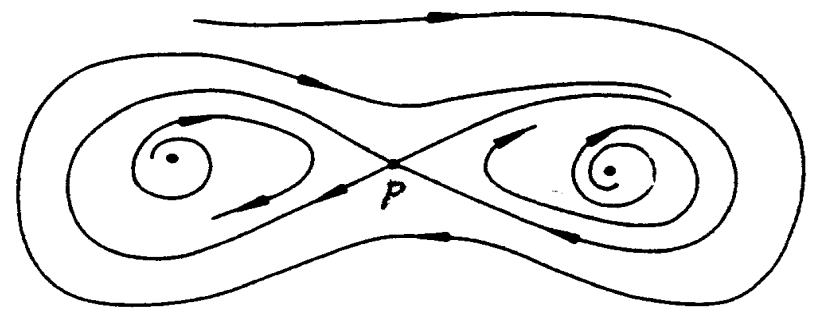

Fig. 1 The figure-eight attractor

\subsection{Attractors with dominated splittings}

A compact $f$-invariant set $\Lambda \subset M$ is called an attractor if there is an open set $U$ with $\Lambda \subset U \subset M$ such that for every $x \in U, d\left(f^{n} x, \Lambda\right) \rightarrow 0$ as $n \rightarrow \infty$. We assume that either $\Lambda=U=M$ or $\overline{f(U)} \subset U$, so that if a perturbation is sufficiently localized, i.e., if the support of $p(\cdot \mid x)$ is contained in $U$ for all $x \in U$, then $\left.\mathcal{X}^{\varepsilon}\right|_{U}$ is a well-defined Markov chain. We observe that under the conditions above, all zero-noise limits of $\left.\mathcal{X}^{\varepsilon}\right|_{U}$ are supported on $\Lambda$. 
Definition 5. A compact $f$-invariant set $\Lambda \subset M$ is said to have a dominated splitting if there exists a $D f$-invariant continuous splitting of the tangent bundle $T \Lambda=E \oplus K$ and constants $0<\tau<1$ and $C \geq 1$ such that for all $x \in \Lambda$,

$$
\frac{\left\|D f^{n}(x) w\right\|}{\|w\|} \leq C \tau^{n} \frac{\left\|D f^{n}(x) v\right\|}{\|v\|}
$$

for all non-zero $w \in K(x)$ and $v \in E(x)$. A shorthand for this is " $\left.D f\right|_{E}>\left.D f\right|_{K}$ ".

Let $f$ and $\Lambda$ be as above, and let $\mu$ be an $f$-invariant Borel probability measure supported on $\Lambda$. We say $(f, \mu)$ has no mixed behavior with respect to the splitting $E \oplus K$ if at $\mu$-a.e. $x, \lambda(x, u) \geq 0$ for all $u \in E(x)$ and $\lambda(x, v) \leq 0$ for all $v \in K(x)$. The triple $(f, \Lambda, \mu)$ with a dominated splitting and no mixed behavior can be thought of as an intermediate class between Axiom A and fully nonuniformly hyperbolic systems.

Theorem B. Suppose $f \in \Omega=\operatorname{Diff}^{\infty}(M)$ has an attractor $\Lambda$ with $T \Lambda=E \oplus K$ and $\left.D f\right|_{E}>\left.D f\right|_{K}$. Then there is a small neighborhood $\mathcal{N}_{1}$ of $f$ in $\operatorname{Diff}^{1}(M)$ for which the following holds: Let $\mathcal{X}^{\varepsilon}$ be such that for all $\varepsilon>0$, $\operatorname{supp}\left(\nu^{\varepsilon}\right) \subset \mathcal{N} \cap \mathcal{N}_{1}$ where $\mathcal{N} \subset \Omega$ is bounded. Let $\left(\mathcal{X}^{\varepsilon}, \mu_{\varepsilon}\right) \rightarrow\left(f, \mu_{0}\right)$, and assume $\mu_{0}$ has no mixed behavior. Then

$$
h_{\mu_{0}}=\lambda_{\mu_{0}}^{+}
$$

We remark that the domination condition does not imply expanding behavior (consider e.g. an attractive fixed point or invariant circle), so one cannot hope to deduce the existence of an SRB measure from the conditions in Theorem B. If $\lambda_{\mu_{0}}^{+}>0$, however, then it follows from Theorem 1 that $\mu_{0}$ or some ergodic component of $\mu_{0}$ is an SRB measure. Results along these lines can be stated without mention of random perturbations. For example:

Corollary 1. Let $f \in \operatorname{Diff}^{\infty}(M)$ be as in Theorem B, i.e., it has an attractor with a dominated splitting $T \Lambda=E \oplus K$. Assume additionally that for every $x \in \Lambda$,

(i) $\liminf _{n \rightarrow \infty} \frac{1}{n} \log \left\|D f_{x}^{n}(v)\right\| \leq 0$ for all $v \in K(x)$,

$\lim \sup _{n \rightarrow \infty} \frac{1}{n} \log \left\|D f_{x}^{n}(v)\right\| \geq 0$ for all $v \in E(x)$;

(ii) $\limsup _{n \rightarrow \infty} \frac{1}{n} \log \left\|\left(\left.D f^{n}\right|_{E}\right)_{x}\right\|>0$.

Then $f$ has an $S R B$ measure.

Corollary 1 is deduced from Theorem B by introducing a small random perturbation of the type required. See $[\mathrm{ABV}]$ for a result in the same direction; the technical hypotheses in $[\mathrm{ABV}]$ and ours differ reflecting the different methods of proof.

We mention next a variant of Theorem $\mathrm{B}$ and two of its corollaries.

Theorem B'. The conclusion of Theorem B holds if its hypotheses are modified in the following way:

(a) $\Omega=\operatorname{Diff}^{\infty}(M)$ is replaced by $\Omega=\operatorname{Diff}^{2}(M)$, and

(b) the additional condition $\inf _{\rho>0} \lim \sup _{\varepsilon \rightarrow 0} h_{\varepsilon, l o c}(\rho)=0$ is imposed on $\mathcal{X}^{\varepsilon}$. 
Corollary 2. Let $f$ be a $C^{2}$ diffeomorphism with a (topologically transitive) Axiom $A$ attractor $\Lambda$. Then

(i) [S1], [R1], [BR] $f$ has an SRB measure $\mu$ supported on $\Lambda$;

(ii) $[\mathrm{K} 1],[\mathrm{Y} 1] \mu$ is stochastically stable for all $\mathcal{X}^{\varepsilon}$ satisfying $(\mathbf{H 1})$ and $(\mathbf{H} 2)$ with $\nu_{\varepsilon}$ supported on a sufficiently small neighborhood $\mathcal{N}$ of $f$ in $\Omega=\operatorname{Diff}^{2}(M)$.

Our second application is to the "almost Anosov maps" studied in [HY]. Let $f$ be a $C^{2}$ diffeomorhism of the 2-torus with the Anosov property except at a single point $p$ fixed by $f$, i.e. $T \mathbf{T}^{2}=E^{c u} \oplus E^{s},\left.D f\right|_{E^{s}}$ is uniformly contracting, and $\left\|\left.D f\right|_{E^{c u}}\right\|>1$ everywhere except at $p$ where $\left\|\left.D f(p)\right|_{E^{c u}}\right\|=1$.

Corollary 3. In the setting of $[\mathrm{HY}]$, the Dirac measure at $p$ is stochastically stable for all $\mathcal{X}^{\varepsilon}$ satisfying $(\mathbf{H 1})$ and $(\mathbf{H 2})$ with $\mathcal{N}$ sufficiently small.

Theorem B' is also used in the proof of Theorem C. The results in Corollary 2 are well known, but our method of proof is new. The result in Corollary 3 is new.

\subsection{Partially hyperbolic attractors with $\operatorname{dim}\left(E^{c}\right)=1$}

We state here a result on the existence of SRB measures proved by introducing small amounts of random noise and letting $\varepsilon \rightarrow 0$.

Definition 6. Let $f$ be a diffeomorphism and $\Lambda$ a compact $f$-invariant set. We say $f$ is partially hyperbolic on $\Lambda$ if there is a splitting of $T \Lambda$ into three (nontrivial) $D f$-invariant subbundles $E^{u u} \oplus E^{c} \oplus E^{s s}$ such that

(i) $\left.D f\right|_{E^{u u}}>\left.D f\right|_{E^{c}}>\left.D f\right|_{E^{s s}}$;

(ii) $\left.D f\right|_{E^{u u}}$ is uniformly expanding and $\left.D f\right|_{E^{s s}}$ is uniformly contracting.

Theorem C. Let $f$ be a $C^{2}$ diffeomorphism with a partially hyperbolic attractor $\Lambda$. If $\operatorname{dim}\left(E^{c}\right)=1$, then $f$ has an $S R B$ measure.

Theorem $\mathrm{C}$ is applicable, for example, to open sets that are neighborhoods of (i) timeone maps of Anosov flows and (ii) products of circle rotations with Axiom A attractors or Anosov diffeomorphisms.

Remark. We stress that in the hypothesis of Theorem $\mathrm{C}$, no restrictions are made on the behavior of $D f$ in the subbundle $E^{c}$. Of particular interest is the situation where $\left\|\left.D f(x)\right|_{E^{c}}\right\|$ is $>1$ for some $x \in \Lambda$ and $<1$ for other $x$, with no a priori information on which behavior is more dominant. Indeed, it may be that neither is dominant, as we allow for the possibility that $\lambda^{c}=0$. This situation is not treated in previous works such as [PeS], $[\mathrm{BV}]$ or $[\mathrm{ABV}]$.

Counterexample with $\operatorname{dim}\left(E^{c}\right)=2$. The statement of Theorem $\mathrm{C}$ is false when $\operatorname{dim}\left(E^{c}\right)>1$. An example is $f=g \times h$ where $g: \mathbb{R}^{2} \rightarrow \mathbb{R}^{2}$ is the time-one-map of the flow in Figure 1 and $h: \mathbb{T}^{2} \rightarrow \mathbb{T}^{2}$ is an Anosov diffeomorphism. Here the attractor of interest is $\Lambda=\Lambda_{1} \times \mathbb{T}^{2}$ where $\Lambda_{1}$ is the figure-eight attractor. If the expansion and contraction of $h$ are sufficiently strong, then $\Lambda$ is partially hyperbolic with $E^{c}$ parallel to 
the first factor $\mathbb{R}^{2}$. Clearly, $f$ has no SRB measures since all invariant probability measures of $f$ are supported on $\{p\} \times \mathbb{T}^{2}$ and have two positive Lyapunov exponents.

Some open questions. Theorem $\mathrm{C}$ suggests or makes more specific a number of questions related to recent developments. In the discussion to follow, the map is assumed to have a partially hyperbolic attractor. Not all of the ideas below are ours.

(1) What additional conditions imply the existence of SRB measures for partially hyperbolic attractors when $\operatorname{dim}\left(E^{c}\right)>1$ ? Is the case $\operatorname{dim}\left(E^{c}\right)=2$ within reach?

(2) If an SRB measure exists - let us assume to begin with that it has no zero Lyapunov exponents and perhaps even that $\operatorname{dim}\left(E^{c}\right)=1$ - under what conditions is it ergodic? Are the ideas of Pugh and Shub (see e.g. [PuS]) on accessibility and stable ergodicity applicable in this context (without volume-preservation)?

(3) Does a "typical" SRB measure in the setting of Theorem C have nonzero Lyapunov exponents? Shub and Wilkinson [SW] and Ruelle [R4] proved recently that for certain specific volume-preserving partially hyperbolic maps with $\operatorname{dim}\left(E^{c}\right)=1$, the zero Lyapunov exponents can be perturbed away. Do these techniques lead to analogous results in the framework considered here?

(4) Since the hypotheses of Theorem C are robust under (deterministic) perturbations, our result gives open sets of attractors with SRB measures. Within connected components of these open sets, it is apparently possible for the unstable dimension to vary. What is the mechanism? Is the scenario in [RW] relevant?

(5) Finally, how do the ideas of differentiation of SRB measures (see e.g. [D] and [R3]) fare for partially hyperbolic attractors in general, and in the context of Theorem $\mathrm{C}$ in particular?

\section{The Main Issues}

This section contains a general discussion of the main issues surrounding the continuity of entropy and Lyapunov exponents as $\varepsilon \rightarrow 0$ for small random perturbations $\mathcal{X}^{\varepsilon}$ of $f$ given by random maps. We assume $\mathcal{X}^{\varepsilon}$ satisfies (H1) and (H2), let $\mu_{\varepsilon}$ be stationary measures of $\mathcal{X}^{\varepsilon}$, and assume $\mu_{\varepsilon} \rightarrow \mu_{0}$. For simplicity of notation, we will write $h_{\varepsilon}$ instead of $h_{\mu_{\varepsilon}}$ and $\lambda_{\varepsilon}^{+}$instead $\lambda_{\mu_{\varepsilon}}^{+}$for $\varepsilon \geq 0$.

\subsection{Four basic relations}

1. Relation between $h_{\varepsilon}$ and $\lambda_{\varepsilon}^{+}$for $\varepsilon>0$. Together with part (ii) of Theorem 1 , the following general result for random dynamical systems lies at the heart of the line of approach in this paper.

Theorem 2. [LY2] Let $\nu$ be a Borel probability measure on $\Omega=\operatorname{Diff}^{2}(M)$ with

$$
\int \log ^{+}\|g\|_{C^{2}} d \nu(g), \quad \int \log ^{+}\left\|g^{-1}\right\|_{C^{2}} d \nu(g)<\infty,
$$

and let $\mu$ be a stationary measure for the process defined by i.i.d. maps with law $\nu$. If $\mu<<m$, then

$$
h_{\mu}=\lambda_{\mu}^{+}
$$


2. Relation between $h_{0}$ and $\lambda_{0}^{+}$. The following result is known as Ruelle's Inequality. It is a general relation not having anything to do with zero-noise limits.

Theorem 3. [R2] Let $g: M \rightarrow M$ be a $C^{1}$ map preserving a Borel probability measure $\mu$. Then

$$
h_{\mu} \leq \lambda_{\mu}^{+}
$$

3. Relation between $\lambda_{\varepsilon}^{+}$and $\lambda_{0}^{+}$. As explained in Section 2.1, Theorem A together with the figure-eight attractor show that $\lambda_{0}^{+}>\limsup _{\varepsilon \rightarrow 0} \lambda_{\varepsilon}^{+}$can and does happen. Jumping in the other direction, however, is impossible.

Proposition 1. With $\Omega=\operatorname{Diff}^{1}(M)$ and under the usual integrability conditions, we have

$$
\lambda_{0}^{+} \geq \limsup _{\varepsilon \rightarrow 0} \lambda_{\varepsilon}^{+} .
$$

Proposition 1 will not be used in the proofs of Theorems A, B or C. It is included for conceptual completeness. A proof is given in Section 3.2.

4. Relation between $h_{\varepsilon}$ and $h_{0}$. We explain how, in principle, $h_{\varepsilon}$ may fail to be either upper semi-continuous or lower semi-continuous as $\varepsilon \rightarrow 0$.

(a) Why we may have $h_{0}>\limsup _{\varepsilon \rightarrow 0} h_{\varepsilon}$ : Suppose for a fixed partition $\alpha, h_{\varepsilon}(\alpha) \approx h_{\varepsilon}$ for all $\varepsilon \geq 0$. We fix $N$ with $\frac{1}{N} H_{\mu_{0}}(\alpha, N) \approx h_{0}(\alpha)$. If $\alpha$ is sufficiently nice, then for all small enough $\varepsilon>0, \frac{1}{N} H_{\mu_{\varepsilon}}(\alpha, N) \approx \frac{1}{N} H_{\mu_{0}}(\alpha, N)$. For fixed $\varepsilon>0$, the subadditivity of $N \mapsto \frac{1}{N} H_{\mu_{\varepsilon}}(\alpha, N)$ may cause $h_{\varepsilon}(\alpha)$ to be smaller than $\frac{1}{N} H_{\mu_{\varepsilon}}(\alpha, N)$.

(b) Why we may have $h_{0}<\liminf _{\varepsilon \rightarrow 0} h_{\varepsilon}$ : Even if for every (fixed) partition $\alpha$, we have $h_{\varepsilon}(\alpha) \approx h_{0}(\alpha)$ for all $\varepsilon$ sufficiently small, $h_{\varepsilon}$ can still jump down as $\varepsilon \rightarrow 0$ because the diameters of the partitions $\alpha_{\varepsilon}$ needed to give $h_{\varepsilon}\left(\alpha_{\varepsilon}\right) \approx h_{\varepsilon}$ may decrease with $\varepsilon$.

Question: Do these scenarios actually occur for reasonable random perturbations?

Proposition 2 gives a condition that prevents (b) from happening; see Sect. 1.4 for the definition of $h_{\varepsilon, l o c}(\rho)$.

Proposition 2. Assume all $\nu^{\varepsilon}$ are supported on a bounded set $\mathcal{N} \subset \operatorname{Diff}^{1}(M)$. If

$$
\inf _{\rho>0} \limsup _{\varepsilon \rightarrow 0} h_{\varepsilon, l o c}(\rho)=0
$$

then

$$
h_{0} \geq \limsup _{\varepsilon \rightarrow 0} h_{\varepsilon} .
$$

A proof is given in Sect. 3.3. 


\subsection{Upper semi-continuity of $\lambda_{\varepsilon}^{+}$at $\varepsilon=0$}

First we fix some notation. For $x \in M$ and $1 \leq k \leq \operatorname{dim} M$, we define

$$
\left\|\wedge^{k} D f(x)\right\|=\sup \left\{\left|\operatorname{det}\left(\left.D f(x)\right|_{V}\right)\right|: V \subset T_{x} M, \operatorname{dim} V=k\right\},
$$

and

$$
\lambda^{(k)}(x)=\lim _{n \rightarrow \infty} \frac{1}{n} \log \left\|\wedge^{k} D f^{n}(x)\right\|
$$

if this limit exists. The following are immediate:

(i) $\lambda^{(k)}(x)$ is well defined $\mu_{0}$-a.e.;

(ii) $\lambda_{0}^{+}=\int \max _{k}\left\{0, \lambda_{0}^{(k)}\right\} d \mu_{0}$; and

(iii) facts analogous to (i) and (ii) hold for random maps.

Proof of Proposition 1: This result is a consequence of the subadditivity of $n \mapsto$ $\int \log \left\|\wedge^{k} D f^{n}\right\|$. The notation " $a \approx b$ " below means " $a$ and $b$ can be made arbitrarily close". The following are the main steps:

(1) First we choose $N$ so that on a set of $\mu_{0}$-measure $\approx 1$,

$$
\Phi_{N}^{(k)}(x):=\frac{1}{N} \log \left\|\wedge^{k} D f^{N}(x)\right\| \approx \lambda^{(k)}(x)
$$

for $k=1, \cdots, \operatorname{dim} M$. Define

$$
\Phi_{N}:=\max \left\{0, \Phi_{N}^{(1)}, \cdots, \Phi_{N}^{(\operatorname{dim} M)}\right\} .
$$

We observe that $\Phi_{N}$ is a continuous function, and that $\lambda_{0}^{+} \approx \int \Phi_{N} d \mu_{0}$ assuming $N$ is sufficiently large.

(2) Given $\delta>0$, choose $\sigma_{0}>0$ small enough that if $\left\|g_{i}-f\right\|_{C^{1}}<\sigma_{0}$, then for all $x \in M$,

$$
\frac{1}{N} \log \left\|\wedge^{k} D g^{N}(x)\right\| \leq \Phi_{N}^{(k)}(x)+\delta
$$

where $g^{N}=g_{N} \circ \cdots \circ g_{1}$ and $N$ is as above.

(3) Next choose $\varepsilon>0$ small enough that

$$
\nu_{\varepsilon}^{N}\left\{\left(\omega_{1}, \ldots, \omega_{N}\right):\left\|\omega_{i}-f\right\|<\sigma_{0}\right\} \approx 1 .
$$

By making this set sufficiently close to full measure, we can, in fact, ensure that for every $x$ and $k$,

$$
\int \frac{1}{N} \log \left\|\wedge^{k} D \omega^{N}(x)\right\| d \nu_{\varepsilon}^{\mathbf{N}} \leq \Phi_{N}^{(k)}(x)+2 \delta .
$$

(4) For $\varepsilon$ as above, we now consider $\left(\mathcal{X}^{\varepsilon}, \mu_{\varepsilon}\right)$. Decompose $M$ into disjoint sets $A_{0} \cup \cdots \cup$ $A_{\operatorname{dim} M}$ where $A_{0}=\left\{\lambda_{\varepsilon}^{+}=0\right\}$ and $A_{k}=\left\{\lambda_{\varepsilon}^{+}=\lambda_{\varepsilon}^{(k)}\right\}, k \geq 1$. Since each $A_{k}$ is invariant under $\mathcal{X}^{\varepsilon}$, we have, for $k \geq 1$,

$$
\begin{aligned}
& \frac{1}{j N} \int_{A_{k}} \log \left\|\wedge^{k} D \omega^{j N}(x)\right\| d\left(\mu_{\varepsilon} \times \nu_{\varepsilon}^{\mathbf{N}}\right) \\
\leq & \frac{1}{j N} \sum_{i=0}^{j-1} \int_{A_{k}} \log \left\|\wedge^{k} D\left(\omega_{(i+1) N} \circ \cdots \circ \omega_{i N+1}\right)\left(\omega^{i N}(x)\right)\right\| d\left(\mu_{\varepsilon} \times \nu_{\varepsilon}^{\mathbf{N}}\right) \\
= & \frac{1}{N} \int_{A_{k}} \log \left\|\wedge^{k} D \omega^{N}(x)\right\| d\left(\mu_{\varepsilon} \times \nu_{\varepsilon}^{\mathbf{N}}\right) .
\end{aligned}
$$


Letting $j \rightarrow \infty$ and summing over $k$, we obtain

$$
\lambda_{\varepsilon}^{+} \leq \int_{M} \Phi_{N} d \mu_{\varepsilon}+2 \delta
$$

This gives the desired result, for $\int \Phi_{N} d \mu_{\varepsilon} \rightarrow \int \Phi_{N} d \mu_{0}$ as $\varepsilon \rightarrow 0$ as $\delta$ can be chosen arbitrarily small.

\subsection{Upper semi-continuity of $h_{\varepsilon}$ at $\varepsilon=0$}

Definition 7. A finite partition $\mathcal{P}$ is $\mu$-regular if each $P \in \mathcal{P}$ has non-empty interior and $\mu(\partial P)=0$. The mesh of $\mathcal{P}$ is $\|\mathcal{P}\|=\max \{\operatorname{diam}(P): P \in \mathcal{P}\}$.

Regular partitions with arbitrarily small mesh may be constructed for any finite measure $\mu$ by choosing appropriate cubes in local charts.

Lemma 4. Suppose $\mu_{\varepsilon} \rightarrow \mu_{0}$ and let $\beta$ be a $\mu_{0}$-regular partition. Then the following hold for any fixed $n \in \mathbf{N}$ and $\beta$ :

(a) given $\delta>0$ there exist $\varepsilon_{0}, \sigma_{0}>0$ such that

$$
\left|\frac{1}{n} H_{\mu_{\varepsilon}}(\omega, \beta, n)-\frac{1}{n} H_{\mu_{0}}(f, \beta, n)\right|<\delta
$$

whenever $\varepsilon<\varepsilon_{0}$ and $\left\|\omega_{i}-f\right\|_{C^{0}}<\sigma_{0}$ for all $i \leq n$;

(b)

$$
\left|\frac{1}{n} H_{\mu_{\varepsilon}}(\beta, n)-\frac{1}{n} H_{\mu_{0}}(f, \beta, n)\right| \rightarrow 0 \quad \text { as } \varepsilon \rightarrow 0 .
$$

Proof: (a) is an easy exercise. To prove (b), we give ourselves an allowed error of $2 \delta$ and choose $\varepsilon_{0}$ and $\sigma_{0}$ as in (a). Let $\mathcal{G}_{n}=\left\{\omega^{n}:\left\|\omega_{i}-f\right\|_{C^{0}}<\sigma_{0} \forall i \leq n\right\}$. Then the difference in (a) integrated over $\mathcal{G}_{n}$ is $<\delta$. We claim the integral over $\mathcal{G}_{n}^{c}$ is also $<\delta$ for $\varepsilon$ sufficiently small. This is because the integrand is uniformly bounded, and $\left(\nu^{\varepsilon}\right)^{n}\left(\mathcal{G}_{n}^{c}\right) \rightarrow 0$ as $\varepsilon \rightarrow 0$.

Proof of Proposition 2: Let $\beta$ be a $\mu_{0}$-regular partition of $M$. For each $n>0$, let

$$
\delta_{n, \beta}=\frac{1}{n} H_{\mu_{0}}(f, \beta, n)-h_{\mu_{0}}(f, \beta) .
$$

Then $\delta_{n, \beta}>0$, and for fixed $\beta, \delta_{n, \beta} \rightarrow 0$ as $n \rightarrow \infty$. Next, we define

$$
\delta_{\varepsilon, n, \beta}^{\prime}=\left|\frac{1}{n} H_{\mu_{\varepsilon}}(\beta, n)-\frac{1}{n} H_{\mu_{0}}(f, \beta, n)\right| .
$$

The previous lemma states that for fixed $\beta$ and $n, \delta_{\varepsilon, n, \beta}^{\prime} \rightarrow 0$ as $\varepsilon \rightarrow 0$.

Altogether we have

$$
\begin{aligned}
h_{\mu_{0}}(f) & \geq h_{\mu_{0}}(f, \beta) \\
& =\frac{1}{n} H_{\mu_{0}}(f, \beta, n)-\delta_{n, \beta} \\
& \geq \frac{1}{n} H_{\mu_{\varepsilon}}(\beta, n)-\delta_{\varepsilon, n, \beta}^{\prime}-\delta_{n, \beta} \\
& \geq h_{\varepsilon}(\beta)-\delta_{\varepsilon, n, \beta}^{\prime}-\delta_{n, \beta},
\end{aligned}
$$


where the last inequality holds because $h_{\varepsilon}(\beta)=\inf _{n} \frac{1}{n} H_{\mu_{\varepsilon}}(\beta, n)$. We first let $\varepsilon \rightarrow 0$ with $\beta$ and $n$ fixed, and then let $n \rightarrow \infty$ to find that

$$
h_{\mu_{0}}(f) \geq \limsup _{\varepsilon \rightarrow 0} h_{\varepsilon}(\beta) .
$$

Now Lemma 3 (with $\mu_{\varepsilon}$ in place of $\mu$ and $\beta$ in place of $\alpha$ ) implies

$$
\limsup _{\varepsilon \rightarrow 0} h_{\varepsilon} \leq \limsup _{\varepsilon \rightarrow 0} h_{\varepsilon}(\beta)+\limsup _{\varepsilon \rightarrow 0} h_{\nu_{\varepsilon}, l o c}(\|\beta\|) .
$$

Substitution of (4) into this inequality yields

$$
\limsup _{\varepsilon \rightarrow 0} h_{\varepsilon} \leq h_{\mu_{0}}+\limsup _{\varepsilon \rightarrow 0} h_{\nu_{\varepsilon}, l o c}(\|\beta\|) .
$$

We write $\rho=\|\beta\|$ and take the infimum over $\rho>0$. The assumption in Proposition 2 is precisely that the term involving $h_{\varepsilon, l o c}:=h_{\nu_{\varepsilon}, l o c}$ vanishes.

\section{Proving $h_{\mu_{0}}=\lambda_{\mu_{0}}^{+}$}

\subsection{A recurring theme}

Suppose $\left(\mathcal{X}^{\varepsilon}, \mu_{\varepsilon}\right) \rightarrow\left(f, \mu_{0}\right)$. The discussion in Section 3 suggests that to show $\mu_{0}$ or an ergodic component of $\mu_{0}$ is an SRB measure, we seek to prove the following relation:

$$
\text { (*) } \quad h_{0} \geq \limsup _{\varepsilon \rightarrow 0} h_{\varepsilon}=\limsup _{\varepsilon \rightarrow 0} \lambda_{\varepsilon}^{+} \geq \lambda_{0}^{+} .
$$

Once we have this, it follows from Ruelle's Inequality (Theorem 3) that $h_{0}=\lambda_{0}^{+}$. If this number is positive, the desired conclusion about $\mu_{0}$ follows by a direct application of Theorem 1 and the remark following it.

For $\mathcal{X}^{\varepsilon}$ satisfying (H1) and (H2), the equality in $(*)$ is always valid by Theorem 2 . Proving $(*)$, therefore, boils down to proving

and

$$
(*)(1) \quad h_{0} \geq \lim \sup _{\varepsilon \rightarrow 0} h_{\varepsilon}
$$

$$
(*)(2) \quad \lim \sup _{\varepsilon \rightarrow 0} \lambda_{\varepsilon}^{+} \geq \lambda_{0}^{+} .
$$

By Proposition 2, (*)(1) is implied by

$$
(*)\left(1^{\prime}\right) \quad \inf _{\rho>0} \lim \sup _{\varepsilon \rightarrow 0} h_{\varepsilon, l o c}(\rho)=0 .
$$

In Sects. 4.2 and 4.3, the scheme of proof summarized above is carried out in two situations. That is to say, we will prove $h_{0}=\lambda_{0}^{+}$by either showing, or assuming, $(*)\left(1^{\prime}\right)$ and $(*)(2)$. 


\subsection{Proof of Theorem A}

Here $(*)(2)$ is part of the hypotheses, and $(*)\left(1^{\prime}\right)$ follows immediately from

Proposition 3. Let $\mathcal{N} \subset \operatorname{Diff}^{\infty}(M)$ be bounded. Then for every $r \in \mathbb{Z}^{+}$, there exists $\rho_{0}(r)>0$ such that for all $\omega=\left(\omega_{1}, \omega_{2}, \cdots\right)$ with $\omega_{i} \in \mathcal{N}$ and $\rho<\rho_{0}(r)$,

$$
h_{l o c}(\omega, \rho) \leq \frac{m}{r} \log R_{1}
$$

where $m=\operatorname{dim}(M)$ and $R_{1}=\sup \{\|D g\|: g \in \mathcal{N}\}$.

To prove Theorem A, then, it remains only to justify Proposition 3.

The corresponding result for the iteration of a single map is known $([\mathrm{Y}],[\mathrm{N}],[\mathrm{Bu}])$. The backbone of this result is a "renormalization theorem" of Yomdin which he used to prove estimates on local growth rates of $\ell$-dimensional volume under the iteration of a map. Newhouse made the connection between volume growth and entropy growth. Then Buzzi observed, insightfully, that a small adaptation of Yomdin's renormalization theorem can be used to give directly, in a very simple argument, a proof of the single-map case of Proposition 3. Buzzi's proof in fact does not make any distinction between random maps and the single map case provided there is a uniform $C^{r}$ bound for all the $\omega_{i}$ (which we have). For completeness, we include below a sketch of the ideas involved, and direct the reader to the respective papers for details.

For the rest of this section $m$ is the dimension of the manifold $M$ and not the Riemannian measure on $M$; the latter will not appear in this discussion.

Let $Q=[-1,1]$, and let $B(a, r) \subset \mathbf{R}^{m}$ be the ball of radius $r$ centered at $a$. We take the liberty to introduce the following language: A $C^{r}$ map $\sigma: Q^{\ell} \rightarrow \mathbf{R}^{m}$ is said to be " $C^{r}$ normalized" if $\|\sigma\|_{C^{r}} \leq 1$. Given $\varphi: Q^{\ell} \rightarrow \mathbf{R}^{m}$ and a set $S \subset \mathbf{R}^{m}$, we say the collection

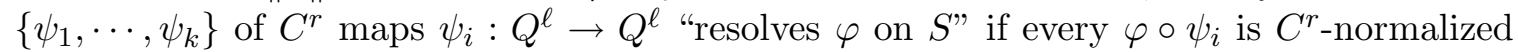
and

$$
S \cap \operatorname{Image}(\varphi) \subset \bigcup_{i=1}^{k} \operatorname{Image}\left(\varphi \circ \psi_{i}\right) .
$$

A resolving collection is easily obtained by choosing a sufficiently large collection of, say, affine contractions. Yomdin proved that a resolving collection can be chosen with cardinality bounded by a quantity which depends only on $r, \ell, m$, and $\|\varphi\|_{C^{r}}$.

Theorem 4 ("Renormalization" Theorem) $[\mathrm{Y}]$ Given a $C^{r}$-normalized map $\sigma: Q^{\ell} \rightarrow$ $B(0,2)$ and $a C^{r}$ map $\varphi: B(0,2) \rightarrow \mathbf{R}^{m}$, the number of $C^{r}$ embeddings $\psi_{i}: Q^{\ell} \rightarrow Q^{\ell}$ needed to resolve $\varphi \circ \sigma$ on $B(0,1)$ is $\leq C_{1}\|\varphi\|_{C^{r}}^{\frac{\ell}{r}}$ where $C_{1}=C_{1}(r, \ell, m)$.

The proof of Proposition 3 requires only the $\ell=m$ case of Theorem 4 . Also, since Theorem 4 will be applied only to maps $\varphi$ which are magnifications of $\omega_{i}$, i.e., $\varphi=\hat{\omega}_{i}:=$ $\chi_{\left(\omega_{i}(x)\right)} \circ \omega_{i} \circ \chi_{x}^{-1}$ where $\chi_{x}: B(x, \rho) \rightarrow B(0,1)$ is the magnification $y \mapsto(y-x) / \rho$, and $\rho$ can be chosen as small as we wish, we may assume that $\left\|D^{s} \varphi\right\|<<1$ for $s \geq 2$ (because $s$-derivatives decrease by a factor of $\rho^{s-1}$ when a map is magnified). In particular, the bound in Theorem 4 may be taken to be $C_{1}\|D \varphi\|^{\frac{\ell}{r}}$. 
Buzzi observed that the $C^{r}$ embeddings $\psi_{i}: Q^{\ell} \rightarrow Q^{\ell}$ in Yomdin's Theorem are contracting, i.e., $d\left(\psi_{i}(x), \psi_{i}(y)\right) \leq d(x, y)$ for all $x, y$. This will be important in the argument to follow.

Proof of Proposition 3 assuming Theorem 4 (following [Bu]): We assume for simplicity that $M=\mathbf{R}^{m}$. For fixed $x_{0} \in \mathbf{R}^{m}$, we have $\hat{\omega}_{i}: B(0,2) \rightarrow \mathbf{R}^{m}, i \geq 1$, where $\hat{\omega}_{i}$ is the magnification of $\omega_{i}$ as explained earlier. Write $\hat{\omega}^{k}=\hat{\omega}_{k} \circ \cdots \circ \hat{\omega}_{1}$ and let $\hat{B}_{n}=\left\{x: \hat{\omega}^{i}(x) \in B(0,1)\right.$ for $\left.i=0,1, \cdots, n\right\}$. The task at hand is to count the number of points needed to $(n, \delta)$-span this set for $\delta$ arbitrarily small.

To this end, we fix a degree of differentiability $r<\infty$, choose $\rho=\rho(r)>0$ so small that $\|\hat{g}\|_{C^{r}}=\|D g\|$ for all $g \in \mathcal{N}$, and let $\kappa=\kappa(r)=C_{1} R_{1}^{\frac{\ell}{r}}$, where $R_{1}$ is as in the statement of the proposition. For each $n \geq 1$, we inductively define a resolving collection $\Psi_{n}$ of $\omega^{n}$, with $\operatorname{card}\left(\Psi_{n}\right) \leq \kappa^{n}$ :

In the first step, we apply Theorem 4 with $\varphi=\hat{\omega}_{1}$ and $\sigma=\sigma_{0}: Q^{m} \hookrightarrow B(0,2)$ the inclusion map, to obtain a collection $\Psi_{1}=\left\{\psi_{i}\right\}$ which resolves $\hat{\omega}_{1} \circ \sigma_{0}$ and has $\operatorname{card}\left(\Psi_{1}\right) \leq \kappa$. Henceforth we regard $\sigma_{0}$ as the identity and repress it.

Suppose, then, that we have a collection $\Psi_{n}$ which resolves $\hat{\omega}^{n}$ and has $\operatorname{card}\left(\Psi_{n}\right) \leq \kappa^{n}$. For each $\psi \in \Psi_{n}$ we apply Theorem 4 with $\varphi=\hat{\omega}_{n+1}$ and $\sigma=\hat{\omega}^{n} \circ \psi$ to obtain a collection $\tilde{\Psi}_{\psi}$ of at most $\kappa$ maps which resolves $\hat{\omega}^{n+1} \circ \psi$ on $B(0,1)$. Our desired collection is

$$
\Psi_{n+1}=\left\{\psi \circ \tilde{\psi} \mid \psi \in \Psi_{n}, \tilde{\psi} \in \tilde{\Psi}_{\psi}\right\} .
$$

Now fix a partition $\mathcal{Q}$ of $Q^{m}$ into sets of diameter $<\delta$, and let $Z$ be a set of representatives, one point from each element of $\mathcal{Q}$. We claim that

$$
\bigcup_{\psi \in \Psi_{n}} \psi(Z)
$$

is $(n, \delta)$-spanning for $\hat{\omega}$. It suffices to show that

$$
d\left(\hat{\omega}^{k} \circ \psi(x), \hat{\omega}^{k} \circ \psi(y)\right) \leq d(x, y), \quad 1 \leq k \leq n,
$$

for every $\psi \in \Psi_{n}$ and $x, y \in Q^{m}$. So let $\psi_{n} \in \Psi_{n}$; by construction, for $1 \leq k \leq n$ there is $\psi_{k} \in \Psi_{k}$ and a (composition of) contraction(s) $\tilde{\psi}_{k, n}: Q^{m} \rightarrow Q^{m}$ such that $\psi_{n}=\psi_{k} \circ \tilde{\psi}_{k, n}$ (with $\tilde{\psi}_{n, n}=$ identity), so

$$
\begin{aligned}
d\left(\hat{\omega}^{k} \circ \psi_{n}(x), \hat{\omega}^{k} \circ \psi_{n}(y)\right) & =d\left(\hat{\omega}^{k} \circ \psi_{k} \circ \tilde{\psi}_{k, n}(x), \hat{\omega}^{k} \circ \psi_{k} \circ \tilde{\psi}_{k, n}(y)\right) \\
& \leq d\left(\tilde{\psi}_{k, n}(x), \tilde{\psi}_{k, n}(y)\right) \leq d(x, y),
\end{aligned}
$$

where we have used that each $\hat{\omega}^{k} \circ \psi_{k}$, being normalized, is also contracting.

We have thus found an $(n, \delta)$-spanning set with cardinality $\leq \kappa^{n} \operatorname{card}(\mathcal{Q})$, hence

$$
h_{l o c}(\omega, \rho) \leq \frac{m}{r} \log R_{1}+\log C_{1} .
$$

To get rid of the $C_{1}$-term, do the usual trick of proving the result above not for $\omega$ but for the sequence $\omega^{(q)}=\left(\omega^{q},\left(\theta^{q} \omega\right)^{q},\left(\theta^{2 q} \omega\right)^{q}, \ldots\right)$ and verifying that

$$
h_{l o c}(\omega, \rho) \leq \frac{1}{q} h_{l o c}\left(\omega^{(q)}, \rho\right) \leq \frac{m}{r} \log R_{1}+\frac{1}{q} \log C_{1} .
$$

Taking $q \rightarrow \infty$ yields $h_{l o c}(\omega, \rho) \leq \frac{m}{r} \log R_{1}$ for all $\omega$ with $\omega_{i} \in \mathcal{N}$ and $\rho<\rho(r)$. 


\subsection{Proofs of Theorem B and Related Results}

Again we follow the scheme in Sect. 4.1. In Theorem B', $(*)\left(1^{\prime}\right)$ is assumed. In Theorem $\mathrm{B},(*)\left(1^{\prime}\right)$ follows from the $C^{\infty}$ assumptions on $f$ and $\mathcal{X}^{\varepsilon}$ and Proposition 3. To complete the proofs of Theorems B and B', then, it suffices to prove $(*)(2)$.

Proposition 4. Let $f$ be a $C^{1}$ diffeomorphism with an attractor on which there is a dominated splitting. If $\operatorname{supp}\left(\nu^{\varepsilon}\right)$ is contained in a $C^{1}$ neighborhood $\mathcal{N}_{1}$ of $f$ that is sufficiently small, then

$$
\limsup _{\varepsilon \rightarrow 0} \lambda_{\varepsilon}^{+} \geq \lambda_{0}^{+} .
$$

For simplicity, we prove our results under the assumption that the domination $\left.D f\right|_{E}>$ $\left.D f\right|_{K}$ occurs in one step, i.e. there exists $\tau<1$ such that for all $x \in \Lambda$ and unit vectors $u \in K(x), v \in E(x)$,

$$
\|D f(x) u\| \leq \tau\|D f(x) v\|
$$

The general case follows easily by considering a power of $f$.

\section{Cone families associated with dominated splittings}

We set some notation and recall some relevant facts. Let $f$ and $\Lambda$ be as in Section 2.2. The dominated splitting on $\Lambda$ implies that for every $r>0$, the family of cones

$$
C^{+}(x, r):=\left\{v=\left(v_{1}, v_{2}\right) \in E(x) \oplus K(x)=T_{x} M:\left|v_{2}\right| \leq r\left|v_{1}\right|\right\}
$$

is $D f$-invariant for all $x \in \Lambda$, meaning $D f(x)\left(C^{+}(x, r)\right) \subset \operatorname{int} C^{+}(f(x), \tau r)$. For any fixed $r>0$ and $\hat{\tau}>\tau$, this invariant cones property is easily extended to a neighborhood $U_{1}$ of $\Lambda$. More precisely, we first continuously extend $E \oplus K$ to a neighborhood $U_{2}$ of $\Lambda$, and point out that this extension need not be invariant on $U_{2} \backslash \Lambda$. Given $r>0$ and $\hat{\tau}>\tau$, we can find $U_{1} \subset U_{2}$ so that $D f(x)\left(C^{+}(x, r)\right) \subset \operatorname{int} C^{+}(f(x), \hat{\tau} r)$ whenever $x$ and $f(x)$ are both in $U_{1}$. By reversing the roles of $f$ and $f^{-1}$, we have similarly a family of $D f^{-1}$-invariant cones $C^{-}(x)$ on $U_{1}$. Moreover, for the same reasons as above, for all maps $g$ sufficiently near $f$ in the $C^{1}$-metric, the cone families above continue to be $D g$ - and $D g^{-1}$-invariant. Having done all this, we write $C^{ \pm}(x)=C^{ \pm}(x, r)$.

Now let $j=\operatorname{dim} E$, and let $\operatorname{Gr}(V, j)$ denote the Grassmannian of $j$-dimensional subspaces in the vector space $V$. We define

$$
C_{E}^{+}(x):=\left\{E^{\prime} \in \operatorname{Gr}\left(T_{x} M, j\right): v \in C^{+}(x) \forall v \in E^{\prime}\right\} .
$$

Then clearly, $D f(x)\left(C^{+}(x)\right) \subset \operatorname{int} C^{+}(f(x))$ implies $D f(x)\left(C_{E}^{+}(x)\right) \subset C_{E}^{+}(f(x))$. Moreover, if we define the distance between $E_{1}$ and $E_{2} \in C_{E}^{+}(x)$ to be $d\left(E_{1}, E_{2}\right)=\left\|L_{1}-L_{2}\right\|$ where $L_{1}, L_{2}: E(x) \rightarrow K(x)$ are the linear maps with $E_{i}=\operatorname{graph}\left(L_{i}\right)$, then (5) implies $d\left(D f(x) E_{1}, D f(x) E_{2}\right)<\hat{\tau} d\left(E_{1}, E_{2}\right)$. It follows that for $x \in \Lambda$ and $n>0$, if $E_{n} \in$ $C_{E}^{+}\left(f^{-n}(x)\right)$ are (arbitrary) trial planes, then $D f^{n}\left(f^{-n}(x)\right) E_{n}$ converges exponentially fast to $E(x)$ as $n \rightarrow \infty$. By considering $f^{-1}$, analogous results hold for the subbundle $K$.

Proof of Proposition 4: Shrinking $U_{1}$ if necessary, we may assume $f\left(\bar{U}_{1}\right) \subset U_{1}$. We require that $\mathcal{N}_{1}$ be small enough that all $g \in \mathcal{N}_{1}$ satisfy $g\left(\bar{U}_{1}\right) \subset U_{1}$ and leave invariant the cone families $\left\{C^{+}\right\}$and $\left\{C^{-}\right\}$. 
Next we introduce the random subbundle $(\omega, x) \mapsto K(\omega, x)$ corresponding to $K$. (Note that $E(\omega, x)$ does not make sense as its definition depends on the "past".) For $\omega=$ $\left(\omega_{1}, \omega_{2}, \cdots\right)$ with $\omega_{i} \in \mathcal{N}_{1}$ and $x \in U_{1}$, we choose for $n>0$ trial planes $K_{n} \in C_{K}^{-}\left(\omega^{n}(x)\right)$ and define

$$
K(\omega, x)=\lim _{n \rightarrow \infty}\left(D \omega^{n}\right)^{-1}\left(\omega^{n}(x)\right) K_{n} .
$$

For the same reasons as in the iteration of a single map, the convergence is exponential, and the limit is well defined and independent of the choice of $K_{n}$. The exponential convergence implies that $K(\omega, x)$ depends continuously on $(\omega, x)$.

Now let $\mathcal{X}^{\varepsilon}$ be such that $\operatorname{supp}\left(\nu_{\varepsilon}\right) \subset \mathcal{N}_{1}$, and define

$$
\lambda_{\varepsilon}^{K}:=\int_{M} \int_{\Omega^{\mathbf{N}}} \log \left|\operatorname{det}\left(\left.D \omega_{1}(x)\right|_{K(\omega, x)}\right)\right| d \nu_{\varepsilon}^{\mathbf{N}} d \mu_{\varepsilon} .
$$

Formula (6) makes sense for $\varepsilon=0$ as well with $\nu_{0}=\delta_{f}$. We observe that $\lambda_{\varepsilon}^{K} \rightarrow \lambda_{0}^{K}$ as $\varepsilon \rightarrow 0$. This is true by the continuity of $(\omega, x) \mapsto \log \left|\operatorname{det}\left(\left.D \omega_{1}(x)\right|_{K(\omega, x)}\right)\right|$ and the convergence of $\nu_{\varepsilon}^{\mathbf{N}} \times \mu_{\varepsilon}$ to $\nu_{0}^{\mathbf{N}} \times \mu_{0}$. A similar (and simpler) argument gives $\lambda_{\varepsilon}^{T M} \rightarrow \lambda_{0}^{T M}$ as $\varepsilon \rightarrow 0$ where

$$
\lambda_{\varepsilon}^{T M}:=\int_{M} \int_{\Omega^{\mathbf{N}}} \log \left|\operatorname{det}\left(D \omega_{1}(x)\right)\right| d \nu_{\varepsilon}^{\mathbf{N}} d \mu_{\varepsilon} .
$$

Clearly, by the ergodic theorem, $\lambda_{\varepsilon}^{T M}$ is the integral of the sum of all the Lyapunov exponents of $\left(\mathcal{X}^{\varepsilon}, \mu_{\varepsilon}\right)\left(\left(f, \mu_{0}\right)\right.$ in the case $\left.\varepsilon=0\right)$ counted with multiplicity. By the same token, $\lambda_{\varepsilon}^{K}$ is the integral of the sum of the Lyapunov exponents for vectors in the random subspace $K$. The "no mixed behavior" assumption on $\mu_{0}$ tells us that $\lambda_{0}^{K}$ is the integral of the sum of all the negative Lyapunov exponents. Thus

$$
\lambda_{0}^{+}=\lambda_{0}^{T M}-\lambda_{0}^{K}=\lim _{\varepsilon \rightarrow 0}\left(\lambda_{\varepsilon}^{T M}-\lambda_{\varepsilon}^{K}\right) \leq \limsup _{\varepsilon \rightarrow 0} \lambda_{\varepsilon}^{+},
$$

the last inequality following from the fact that the sum of any subset of Lyapunov exponents is no greater than the sum of the positive ones.

Proof of Corollary 2: SRB measures are constructed by introducing a small random perturbation and using Theorem B'. Observe that $h_{l o c}(\omega, \rho)=0$ for all $\rho<\rho_{0}(f)$ if all the $\omega_{i}$ are $C^{1}$ sufficiently near $f$. In fact, the set $B^{+}(x, \omega, \rho)$ (see Sect. 1.4) is contained in the local stable manifold $W_{2 \rho}^{s}(\omega, x)$. This argument shows that every zero-noise limit is an SRB measure. Stochastic stability follows from the uniqueness of SRB measure on topologically transitive Axiom A attractors.

Proof of Corollary 3: First suppose $f$ is $C^{\infty}$. By Theorem B, every zero-noise limit satisfies $h_{\mu_{0}}=\lambda_{\mu_{0}}^{+}$. This relation holds for $\delta_{p}$, the Dirac measure at $p$; it suffices to show that it is satisfied by no other invariant measure. Suppose, to derive a contradiction, that it is satisfied by $\mu \neq \delta_{p}$. Then $\mu(M \backslash O)>0$ for some small neighborhood $O$ of $p$. Now $|D f|_{E^{c u}} \mid \geq c>1$ outside of $O$, which implies that $\lambda_{\mu}^{+}>0$. By Theorem 1(ii), $\mu$ or some component of $\mu$ is an SRB measure, but it is proved in [HY] that $f$ admits no (finite) SRB measures. 
If $f$ is only $C^{2}$, Theorem B' applies provided the local entropy assumption can be verified. It is in fact true that for the map $f$ in question, $h_{\varepsilon, l o c}(\rho)=0$ for all sufficiently small $\rho$. Even though the sign of the larger Lyapunov exponent for the sequence $\omega$ is unclear, one-dimensional $W^{s s}$-curves are well defined on all of $M$, and $B^{+}(x, \omega, \rho)$ is either contained in $W_{2 \rho}^{s s}(\omega, x)$ or in a one-parameter family of $W_{2 \rho}^{s s}(\omega, \cdot)$-curves. In the latter case, local growth in complexity is at most linear. Since this is a simple case of Proposition 6 (with $E^{u u}=\{0\}$ ), details will not duplicated here.

\section{Proof of Theorem C}

\subsection{The main ideas}

The following notation is used throughout: $E^{c u}:=E^{u u} \oplus E^{c}, E^{c s}:=E^{c} \oplus E^{s s}$. Standard uniform hyperbolic theory informs us that invariant manifolds tangent to $E^{u u}$ and $E^{s s}$ exist. We call them $W^{u u_{-}}$and $W^{s s}$-manifolds.

Theorem $\mathrm{C}$ asserts that partially hyperbolic attractors with $\operatorname{dim}\left(E^{c}\right)=1$ have $\mathrm{SRB}$ measures. We begin by explaining why the usual Axiom A construction may not be adequate here. In this standard construction, one pushes forward Lebesgue measure on a piece of local unstable manifold. Since uniform expansion is needed, and we only have knowledge of uniform expansion on $W^{u u}$-leaves, this construction gives only an invariant measure $\mu$ with absolutely continuous conditional measures on $W^{u u}$-manifolds. If the Lyapunov exponent of $(f, \mu)$ in the $E^{c}$-direction is $\leq 0 \mu$-a.e., then $\mu$ is an SRB measure by definition; otherwise information in the $E^{c}$-direction is needed. This information is not available from either our hypothesis or the above construction.

As announced in the introduction, we will obtain an SRB measure for $f$ as (an ergodic component of) a zero-noise limit. To this end, we choose a small perturbation $\mathcal{X}^{\varepsilon}$ of $f$ such that $\operatorname{supp}\left(\nu^{\varepsilon}\right) \subset \mathcal{N}$ where $\mathcal{N} \subset \mathcal{N}_{1} \cap \mathcal{N}_{2}: \mathcal{N}_{2}$ here can be any bounded subset of $\operatorname{Diff}^{2}(M)$; $\mathcal{N}_{1}$ is a sufficiently small $C^{1}$-neighborhood of $f$. For reasons to become clear in Sect. 5.2, we require further that for each $\varepsilon>0$, the density of $p^{\varepsilon}(\cdot \mid x)$ is strictly positive on the $\varepsilon$-disk centered at $f(x)$ for every $x$. Such perturbations are easily constructed (see the beginning of Section 2).

The two main ingredients of our proof are:

Proposition 5. Every zero-noise limit $\mu_{0}$ of $\mathcal{X}^{\varepsilon}$ has absolutely continuous conditional measures on $W^{u u}$-leaves.

Proposition 6. For all small enough $\rho>0, h_{l o c}(\omega, \rho)=0$ for all $\omega \in \mathcal{N}^{\mathbf{N}}$.

We note that Proposition 6 hinges on the fact that $\operatorname{dim}\left(E^{c}\right)=1$, although this is not the only place where this assumption is used.

Proof of Theorem $\mathbf{C}$ assuming Propositions 5 and 6: Let $\mu_{0}$ be a zero-noise limit of $\mathcal{X}^{\varepsilon}$ where $\mathcal{X}^{\varepsilon}$ is as above. From Proposition 5 , it follows that if $A:=\{x: \lambda(x, v) \leq$ $\left.0 \forall v \in E^{c}(x)\right\}$ has positive $\mu_{0}$-measure, then we are done, for $\mu=\left.\mu_{0}\right|_{A}$ normalized is an SRB measure. We assume therefore that $f$ has a positive Lyapunov exponent in the $E^{c}$ direction $\mu_{0}$-a.e. Letting $E=E^{c u}$ and $K=E^{s s}$, we now find ourselves in the setting of Theorem B', with $\left(f, \mu_{0}\right)$ having no mixed behavior with respect to $E \oplus K$ and with the 
zero local entropy property $(*)\left(1^{\prime}\right)$ given by Proposition 6 . Since $\mu_{0}$ has positive Lyapunov exponents by definition, we conclude that it is an SRB measure.

As to how small a neighborhood of $f$ the set $\mathcal{N}_{1}$ has to be, for a start we need to be able to choose an open set $U_{1}$ with $\Lambda \subset U_{1} \subset M$ such that for all $g \in \mathcal{N}_{1}, g\left(\bar{U}_{1}\right) \subset U_{1}$ and $D g$ and $D g^{-1}$ leave invariant cone families that extend those on $\Lambda$. More precisely, $D g$ is required to preserve cone families $C^{u u}$ and $C^{c u}$, which are defined analogously to $C^{+}$in Section 4 , with $E=E^{u u}$ and $K=E^{c s}$ for $C^{u u}$, and $E=E^{c u}$ and $K=E^{s s}$ for $C^{c u}$. Similarly, $D g^{-1}$ is required to preserve $C^{c s}$ and $C^{s s}$. Lemmas 6 and 7, which contain stable-manifolds type estimates, may require that we shrink $\mathcal{N}_{1}$ and $U_{1}$ further.

\subsection{Conditional measures of $\mu_{0}$ on $W^{u u}$}

Suppose $\left(\mathcal{X}^{\varepsilon}, \mu_{\varepsilon}\right) \rightarrow\left(f, \mu_{0}\right)$. To prove that $\mu_{0}$ has absolutely continuous conditional measures on $W^{u u}$, we show that $\mu_{\varepsilon}$, or, more accurately, the inverse limit of $\mu_{\varepsilon}$, has this property for every $\varepsilon>0$, and then let $\varepsilon \rightarrow 0$. The following is the two-sided version of the construction in Section 1.3.

Let $\theta: \Omega^{\mathbf{Z}} \rightarrow \Omega^{\mathbf{Z}}$ be the shift operator, and define $F: \Omega^{\mathbf{Z}} \times M \rightarrow \Omega^{\mathbf{Z}} \times M$ by

$$
F(\omega, x)=\left(\theta(\omega), \omega_{1}(x)\right)
$$

where $\omega=\left(\cdots, \omega_{-1}, \omega_{0}, \omega_{1}, \omega_{2} \cdots\right)$. Given $\omega$, we let $\omega^{(+)}=\left(\omega_{1}, \omega_{2}, \cdots\right)$ and $\omega^{(-)}=$ $\left(\cdots, \omega^{-1}, \omega_{0}\right)$ denote the future and past of $\omega$ respectively. For $n>0$, we write $\omega^{n}=$ $\omega_{n} \circ \cdots \circ \omega_{1}$ and $\omega^{-n}=\omega_{-(n-1)}^{-1} \circ \cdots \circ \omega_{0}^{-1}$.

The constructions below are reminiscent of those in [Y1].

Proof of Proposition 5: Corresponding to each $\omega \in \mathcal{N}^{\mathbf{Z}}$, we write $M_{\omega}:=\{\omega\} \times M$, and let $\Lambda_{\omega} \subset M_{\omega}$ be given by

$$
\Lambda_{\omega}:=\bigcap_{n \geq 0}\left(\theta^{-n} \omega\right)^{n}\left(\bar{U}_{1}\right) .
$$

Then at each $x \in \Lambda_{\omega}, E^{u u}(\omega, x) \subset T_{x} M$ is well defined, with $E^{u u}(\omega, x)$ varying continuously

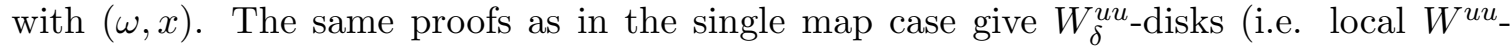
manifolds of radius $\delta$ ) with uniform estimates at every $\omega \in \mathcal{N}^{\mathbf{Z}}$ and $x \in \Lambda_{\omega}$. Global $W^{u u_{-}}$ manifolds are defined accordingly. We have on each $M_{\omega}$, therefore, a "random attractor" laminated by "random $W^{u u}$-manifolds". Note that both $\Lambda_{\omega}$ and its $W^{u u}$-leaves depend only on $\omega^{(-)}$.

To make precise the idea of a measure on $\mathcal{N}^{\mathbf{Z}} \times M$ having absolutely continuous conditional measures on $W^{u u}$-leaves, we introduce the idea of a canonical neighborhood in $\bigcap_{n \geq 0} F^{n}\left(\mathcal{N}^{\mathbf{Z}} \times M\right)$. Let $\Sigma \subset M$ be an embedded disk having dimension $\operatorname{dim}\left(E^{c s}\right)$ and uniformly transverse to the $W^{u u}$-leaves on every $M_{\omega}$. For $\delta>0$, let

$$
\mathcal{U}=\mathcal{U}_{\Sigma, \delta}:=\left\{(\omega, y) \in \mathcal{N}^{\mathbf{Z}} \times M: y \in W_{\delta}^{u u}(\omega, x) \text { for some } x \in \Lambda_{\omega} \cap \Sigma\right\} .
$$

The set $\mathcal{U}$, then, is partitioned measurably by $W_{\delta}^{u u}$-disks. Let $\mu$ be a measure on $\Omega^{\mathbf{Z}} \times M$ with $\mu(\mathcal{U})>0$. We let $\left(\left.\mu\right|_{\mathcal{U}}\right)^{u u}$ denote the conditional measures of $\left.\mu\right|_{\mathcal{U}}$ with respect to this partition, and let $m^{u u}$ denote the measure corresponding to the Riemannian volume on 
$W^{u u}$-disks. The precise meaning of the statement " $\mu$ has absolutely continuous conditional measures $(a c c m)$ on $W^{u u}$-leaves" is that for every canonical neighborhood $\mathcal{U}$ with $\mu(\mathcal{U})>0$, $\left(\left.\mu\right|_{\mathcal{U}}\right)^{u u}$ is absolutely continuous with respect to $m^{u u}$ on the $W_{\delta}^{u u}$-disk containing $\mu$-a.e. $(\omega, x)$. Let $q$ be the densities of $\left(\left.\mu\right|_{\mathcal{U}}\right)^{u u}$ with respect to $m^{u u}$. We say $q$ has uniform bounds if there exists $C$ depending only on $f$ and on $\delta$ such that $C^{-1} \leq q \leq C$ for every $\mathcal{U}$ as above.

The bulk of the work in proving Proposition 5 is contained in the following lemma. Let $\pi_{\Omega^{\mathbf{Z}}}: \Omega^{\mathbf{Z}} \times M \rightarrow \Omega^{\mathbf{Z}}$ and $\pi_{M}: \Omega^{\mathbf{Z}} \times M \rightarrow M$ be projection maps.

Lemma 5. Given $\varepsilon>0$ and a stationary measure $\mu_{\varepsilon}$ of $\mathcal{X}^{\varepsilon}$, there is an F-invariant Borel probability measure $\sigma_{\varepsilon}$ on $\Omega^{\mathbf{Z}} \times M$ with the following properties:

(1) $\sigma_{\varepsilon}$ has accm on $W^{u u}$-leaves with uniform density bounds;

(2) $\left(\pi_{\Omega} \mathbf{z}\right)_{*}\left(\sigma_{\varepsilon}\right)=\nu_{\varepsilon}^{\mathbf{Z}}$ and $\left(\pi_{M}\right)_{*}\left(\sigma_{\varepsilon}\right)=\mu_{\varepsilon}$.

We first finish the proof of Proposition 5 assuming Lemma 5 . Since $\left(\mathcal{X}^{\varepsilon}, \mu_{\varepsilon}\right) \rightarrow\left(f, \mu_{0}\right)$, we have, passing to a subsequence if necessary, $\sigma_{\varepsilon} \rightarrow \sigma_{0}$ for some $\sigma_{0}$. Hence $\left(\pi_{M}\right)_{*}\left(\sigma_{0}\right)=\mu_{0}$, and $\sigma_{0}$ is supported on the fiber $\omega=(\ldots, f, f, f, \ldots)$. The uniform density bounds for the conditional measures on $W^{u u}$-leaves for $\sigma_{\varepsilon}$ are passed to $\sigma_{0}$. This is equivalent to $\mu_{0}$ having accm on the $W^{u u}$-leaves of $f$.

Proof of Lemma 5: By our impositions on $p^{\varepsilon}(\cdot \mid x), \mu_{\varepsilon}$ has at most a finite number of ergodic components living on disjoint open sets. Since the argument below is carried out for one ergodic component of $\mu_{\varepsilon}$ at a time, we may as well assume $\mu_{\varepsilon}$ is ergodic.

Fix a past $\hat{\omega}^{(-)}=\left(\cdots, \hat{\omega}_{-1}, \hat{\omega}_{0}\right)$ with $\hat{\omega}_{i} \in \mathcal{N}$, and remembering that $W_{\delta}^{u u}$-disks on $M_{\omega}$ are determined entirely by $\omega^{(-)}$, we let $\gamma$ be a $W_{\delta}^{u u}$-disk on $M_{\omega}$ where $\omega^{(-)}=\hat{\omega}^{(-)}$. We choose $\gamma$, in fact, so that $\left(\pi_{M}\right)_{*}(\gamma)$ lies in the interior of the support of $\mu_{\varepsilon}$. Let $m_{\gamma}$ denote the normalized Riemannian measure on $\gamma$. We push forward using $F$ the measure

$$
\sigma_{0}^{\varepsilon}:=\left(\delta_{\hat{\omega}(-)} \times \nu_{\varepsilon}^{\mathbf{N}}\right) \times m_{\gamma}
$$

where $\delta_{\hat{\omega}(-)}$ is the Dirac measure on $\Omega^{\mathbf{Z} \backslash \mathbf{N}}$ concentrated at the sequence $\hat{\omega}^{(-)}$. More precisely, we let

$$
\sigma_{n}^{\varepsilon}=\frac{1}{n} \sum_{i=0}^{n-1} F_{*}^{i}\left(\sigma_{o}^{\varepsilon}\right),
$$

and let $\sigma_{\varepsilon}$ be a limit point of $\sigma_{n}^{\varepsilon}$ as $n \rightarrow \infty$. It is easy to see that $\left(\pi_{\Omega^{\mathbf{z}}}\right)_{*}\left(\sigma_{\varepsilon}\right)=\nu_{\varepsilon}^{\mathbf{Z}}$.

To see that $\sigma_{\varepsilon}$ has accm on $W^{u}$-manifolds, we observe that this property is enjoyed by $F_{*}^{n}\left(\sigma_{o}^{\varepsilon}\right)$ for all $n>0$ (the proof is the same as that for a single map; it uses the bounded distortion of $D \omega_{i}$ along $W^{u u}$-leaves, a property guaranteed by uniform expansion and the presence of a uniform bound on $C^{2}$ norms). These uniform density bounds are therefore valid for each $\sigma_{n}^{\varepsilon}$ and passed on to $\sigma_{\varepsilon}$.

To show that $\left(\pi_{M}\right)_{*}\left(\sigma_{\varepsilon}\right)=\mu_{\varepsilon}$, we first prove that $\left(\pi_{M}\right)_{*}\left(\sigma_{\varepsilon}\right)$ is an invariant measure for $\mathcal{X}^{\varepsilon}$. To prove the invariance of $\left(\pi_{M}\right)_{*}\left(\sigma_{\varepsilon}\right)$ is equivalent to showing that it projects onto a product measure on $\Omega^{\mathbf{N}} \times M$ (see Lemma 2 in Section 1.3), or, equivalently, that its conditional measures on $M_{\omega}$ are independent of $\omega^{(+)}$. We claim that this is true by design: for each $n>0, F_{*}^{n}\left(\sigma_{o}^{\varepsilon}\right)$ lives on $M_{\omega}$-fibers with the property that $\omega_{i}=\hat{\omega}_{i+n+1}$ for all $i \leq-n$. On each such $M_{\omega}$, the conditional measure of $F_{*}^{n}\left(\sigma_{o}^{\varepsilon}\right)$ is $\left(\omega_{0} \circ \omega_{-1} \circ \cdots \circ \omega_{-n}\right)_{*}\left(m_{\gamma}\right)$, which evidently does not depend on $\omega^{(+)}$. 
It remains to show that $\left(\pi_{M}\right)_{*}\left(\sigma_{\varepsilon}\right)=\mu_{\varepsilon}$ and not some other invariant measure of $\mathcal{X}^{\varepsilon}$. This is true because $\left(\pi_{M}\right)_{*}\left(F_{*}^{i}\left(\sigma_{0}^{\varepsilon}\right)\right)$ is the distribution of the $i$ th step of $\mathcal{X}^{\varepsilon}$ if the initial distribution is normalized Lebesgue measure on $\left(\pi_{M}\right)_{*}(\gamma)$.

\subsection{Local entropy is zero when $\operatorname{dim} E^{c}=1$}

It remains to show that if $\mathcal{N}$ is a sufficiently small neighborhood of $f$, then there exists $\rho^{*}>0$ such that $h_{\text {loc }}\left(\omega, \rho^{*}\right)=0$ for all $\omega \in \mathcal{N}^{\mathbf{N}}$. The idea of our proof is as follows: For $\rho$ sufficiently small, we show that $B^{+}(x, \omega, \rho)$ is contained in an embedded disk through $x$ having the dimension of $E^{c s}$ and roughly parallel to it. Moreover, since $E^{c}$ is one-dimensional, the $W^{s s}$ leaves which foliate this submanifold are linearly ordered, and application of $\omega_{i}$ preserves this ordering. With $f$ contracting on $W^{s s}$-leaves, we show that the action resembles that of an interval homeomorphism, and hence the growth in complexity is at most linear.

Proof of Proposition 6: First we fix some notation. Let $U_{1}$ be as at the end of Sect. 5.1. For $x \in U_{1}$, let $E_{\rho}^{u u}(x)$ denote the closed disk of radius $\rho$ in $E^{u u}(x) ; E_{\rho}^{c}(x)$ and $E_{\rho}^{s s}(x)$ are defined similarly. Let $E_{\rho}(x)=E_{\rho}^{u u}(x) \times E_{\rho}^{c}(x) \times E_{\rho}^{s s}(x)$, and let $\exp _{x}: T_{x} M \rightarrow M$ be the exponential map at $x$. We fix $\rho_{0}$ small enough for purposes of Lemmas 6 and 7 below. For $g \in \mathcal{N}$ and $x \in U_{1}$, let

$$
\tilde{g}_{x}:=\exp _{g x}^{-1} \circ g \circ \exp _{x}: E_{\rho_{0}}(x) \rightarrow T_{g x} M .
$$

The arguments in this proof are entirely local. We will, for each pair $(x, \omega)$, work exclusively in the charts $\left\{E_{\rho_{0}}\left(\omega^{j} x\right)\right\}_{j=0,1,2, \cdots}$ via the chart maps $\left(\tilde{\omega}_{j}\right)_{\omega^{j-1} x}: E_{\rho_{0}}\left(\omega^{j-1} x\right) \rightarrow T_{\omega^{j} x} M$. With the metrics in these charts being uniformly equivalent to the Riemannian metric on $M$, it suffices to prove our zero local entropy result for $\left(\tilde{\omega}^{j}\right)_{x}$. For simplicity of notation, then, we will henceforth identify $E_{\rho_{0}}\left(\omega^{j} x\right)$ with $\exp _{\omega^{j} x}\left(E_{\rho_{0}}\left(\omega^{j} x\right)\right)$ and drop the tildes.

We begin with the following technical lemma.

Lemma 6. There exist $\rho_{1}$ and $\rho^{*}$ with $0<\rho^{*}<\rho_{1}<\rho_{0}$ for which the following hold for all $x \in U_{1}$ and $\omega \in \mathcal{N}^{\mathbf{N}}$. Let $(x, \omega)$ be fixed. Then for each $j \in\{0,1,2, \cdots\}$ :

(a) (Local $W^{c s}$-manifolds) There exists $\varphi_{j}: E_{\rho_{1}}^{c s}\left(\omega^{j} x\right) \rightarrow E_{\rho_{1}}^{u u}\left(\omega^{j} x\right)$ with $\varphi_{j}(0)=0$ and $\left\|D \varphi_{j}\right\|<\frac{1}{10}$ so that if $\Sigma_{j}$ is the graph of $\varphi_{j}$, then

(i) $B^{+}\left(x, \omega, \rho^{*}\right) \subset \Sigma_{0}$;

(ii) $\omega_{j}\left(\Sigma_{j-1}\right) \cap E_{\rho_{1}}\left(\omega^{j} x\right) \subset \Sigma_{j}$.

(b) (Local $W^{s s}$-manifolds) For all $y \in B^{+}\left(x, \omega, \rho^{*}\right)$, there exists $\psi_{y}: E_{\rho_{1}}^{s s}(x) \rightarrow E_{\rho_{1}}^{c u}(x)$ with $\left\|D \psi_{y}\right\|<\frac{1}{10}$ such that

(i) $W_{l o c}^{s s}(y) \cap E_{\rho_{1}}(x)=\operatorname{graph}\left(\psi_{y}\right):=W_{\rho_{1}}^{s s}(y)$;

(ii) $\omega^{j}\left(W_{\rho_{1}}^{s s}(y)\right) \subset\left(\Sigma_{j} \cap E_{\rho_{1}}\left(\omega^{j} x\right)\right) \quad \forall j \geq 0$.

We omit the proof of this lemma, which follows from standard graph transform arguments. Let

$$
\hat{B}(x, \omega)=\bigcup_{y \in B^{+}\left(x, \omega, \rho^{*}\right)} W_{\rho_{1}}^{s s}(y) .
$$


Notice that (i) $\hat{B}(x, \omega) \supset B^{+}\left(x, \omega, \rho^{*}\right)$, so it suffices to bound the cardinality of $(n, \delta)$ spanning sets on $\hat{B}(x, \omega)$; (ii) geometrically, $\hat{B}(x, \omega)$ is the disjoint union of $W_{\rho_{1}}^{\text {ss }}$-disks; and (iii) $\omega^{j}(\hat{B}(x, \omega)) \subset \Sigma_{j}$ for all $j$, so its future iterates are well controlled.

Let $\gamma=E_{\rho_{1}}^{c u}(x) \cap \Sigma_{0}$. Then $\gamma$ is a curve that meets every $W_{\rho_{1}}^{s s}$-disk in $\tilde{B}(x, \omega)$ in exactly one point. Let $\tilde{\gamma}=\gamma \cap \tilde{B}(x, \omega)$, and fix arbitrary $\delta>0$. Let $r(\delta, n, K)$ denote the minimal cardinality of an $(n, \delta)$-spanning set in $K$ (see Sect. 1.4). Then

$$
r(\delta, n, \tilde{B}(x, \omega)) \leq C\left(\frac{\rho_{1}}{\delta}\right)^{k} r(\delta, n, \tilde{\gamma}) \quad \text { where } \quad k=\operatorname{dim} E^{s s} .
$$

The problem is thus reduced to estimating $r(\delta, n, \tilde{\gamma})$.

Our next lemma gives control of the geometry of $\omega^{j}(\tilde{\gamma})$ for all $j \geq 0$. For $y \in E_{\rho_{0}}$, let $y=\left(y^{u u}, y^{c}, y^{s s}\right)$ be the coordinates with respect to $E^{u u} \oplus E^{c} \oplus E^{s s}$.

Lemma 7. For $y, z \in \gamma$ and $n \in \mathbf{N}$, if $\omega^{j} y, \omega^{j} z \in E_{\rho_{1}}\left(\omega^{j} x\right)$ for all $j \leq n$, then

(i) $\left\|\left(\omega^{n} y-\omega^{n} z\right)^{u u}\right\|<\frac{1}{10}\left\|\left(\omega^{n} y-\omega^{n} z\right)^{c}\right\|$;

(ii) $\left\|\left(\omega^{n} y-\omega^{n} z\right)^{s s}\right\|<\frac{1}{10}\left\|\left(\omega^{n} y-\omega^{n} z\right)^{c}\right\|$.

(i) follows from the fact that $\omega^{n} y, \omega^{n} z \in \Sigma_{n}$, and $\left\|D \varphi_{n}\right\|<\frac{1}{10}$ (see Lemma 6(a)). (ii) is proved inductively using the fact that $\left.D f\right|_{E^{c}}$ dominates $\left.D f\right|_{E^{s s}}$.

From Lemma 7, we see that $\tilde{\gamma}$ is either a point (in which case we are done) or it is a connected curve segment with the property that for all $j \geq 0, \omega^{j}(\tilde{\gamma})$ has length $\leq 2 \rho_{1}$. To estimate $r(\delta, n, \tilde{\gamma})$, we put $\frac{2 \rho_{1}}{\delta}$ points, evenly spaced, along the length of each $\omega^{j}(\tilde{\gamma})$, and let $S_{n}$ be the union of the pull-back to $\tilde{\gamma}$ of all the points on $\omega^{j}(\tilde{\gamma}), j \leq n$. Then $S_{n}$ is clearly an $(n, \delta)$-spanning set for $\tilde{\gamma}$, and its cardinality is $\frac{2 \rho_{1}}{\delta} n$.

\section{References}

[ABV] J.F. Alves, C. Bonatti and M. Viana, SRB measures for partially hyperbolic systems whose central direction is mostly expanding, Invent. Math. 140 (2000), 351-398.

[BV] C. Bonatti and M. Viana, SRB measures for partially hyperbolic systems whose central direction is mostly contracting, Israel J. Math. 115 (2000), 157-194.

[Bo] R. Bowen, Entropy-expansive maps, Transactions of the AMS, 164 (1972), 323-331.

[BR] R. Bowen and D. Ruelle, The ergodic theory of Axiom A flows, Invent. Math. 29 (1975) 181-202.

[Bu] J. Buzzi, Intrinsic ergodicity of smooth interval maps, Israel J of Math., 100 (1997), 125-161.

[D] D. Dolgopyat, On differentiability of SRB states, preprint.

[ER] J.-P. Eckmann and D. Ruelle, Ergodic theory of chaos and strange attractors, Rev. Mod. Phys. 57 (1985) 617-656 
[HY] H. Hu and L.-S. Young, Nonexistence of SRB measures for some systems that are "almost Anosov", Ergodic Theory and Dynamical Systems 15 (1995), 67-76.[HY]

[K1] Yu. Kifer, On small random perturbations of some smooth dynamical systems, Math. USSR Ivestija 8 (1974), 1083-1107.

[K2] Yu. Kifer, Ergodic theory of random transformations, Birkhauser (1986).

[K3] Yu. Kifer, Random Perturbations of Dynamical Systems, Birkhauser, (1988).

[KY] Y. Kifer and Y. Yomdin, Volume growth and topological entropy for random transformations, Lecture Notes in Math., Springer-Verlag, (1988) 361-373.

[L] F. Ledrappier, Propriétés ergodiques des mesures de Sinai, Publ. Math., Inst. Hautes Etud. Sci. 59 (1984), 163-188.

[LS] F. Ledrappier and J.-M. Strelcyn, A proof of the estimation from below in Pesin entropy formula, Ergod. Th. \& Dynam. Sys., 2 (1982) 203-219.

[LY1] F. Ledrappier and L.-S. Young, The metric entropy of diffeomorphisms, Ann. Math. $122(1985)$ 509-574.

[LY2] F. Ledrappier and L.-S. Young, Entropy formula for random transformations, Prob. Th. Rel. Fields 80 (1988) 217-240.

[LQ] P.-D. Liu and M. Qian, Smooth Ergodic Theory of Random Dynamical Systems, Lecture Notes in Math., Springer-Verlag, Vol. 1606 (1995).

[N] S. Newhouse, Continuity properties of entropy, Ann. Math. 129 (1989), 215-235.

[O] V.I. Oseledec, A multiplicative ergodic theorem: Liapunov characteristic numbers of dynamical systems, Trans. Moscow Math. Soc. 19 (1968) 197-231.

[P] Ya. B. Pesin, Characteristic Lyapunov exponents and smooth ergodic theory, Russ. math. Surv. 32.4 (1977), 55-114.

[PeS] Ya. B. Pesin and Ya. G. Sinai, Gibbs measures for partially hyperbolic attractors, Erg. Th. \& Dynam. Sys. 2 (1982) 417-438.

[PuS] C. Pugh and M. Shub, Stable ergodicity and julienne quasi-conformality, J Eur. Math. Soc. (2001).

[R1] D. Ruelle, A measure associated with Axiom A attractors, Amer. J. Math. 98 (1976), 619-654.

[R2] D. Ruelle, An inequality of the entropy of differentiable maps, Bol. Sc. Bra. Mat. 9 (1978) 83-87.

[R3] D. Ruelle, Differentiation of SRB states, Commun. Math. Phys. 187 (1997), 227-241. Differentiation of SRB states: correction and complements, Commun. Math. Phys. 234 (2003) 185-190. 
[R4] D. Ruelle, Perturbation theory for Lyapunov exponents of a toral map: extension of a result of Shub and Wilkinson, Israel J. Math., 134 (2003), 345-361.

[RW] D. Ruelle and A. Wilkinson, Absolutely singular dynamical foliations, Commun. Math. Phys. 219 (2001), 481-487.

[S1] Ya. G. Sinai, Gibbs measure in ergodic theory, Russian Math. Surveys 27 (1972), 21-69.

[S2] Ya. G. Sinai, Kolmogorov's work on ergodic theory, Ann. Prob., 17(3) (1989), 833-839.

[SW] M. Shub and A. Wilkinson, Pathological foliations and removable exponents, Invent. Math. 139, (2000) 495-508.

[Y] Y. Yomdin, Volume growth and entropy, Israel J Math., 57 (1987) 285-300.

[Y1] L.-S. Young, Stochastic stability of hyperbolic attractors, Erg. Th. \& Dynam. Sys. 6 (1986), 311-319.

[Y2] L.-S. Young, What are SRB measures, and which dynamical systems have them? J. Stat. Phys. 108 (2002), 733-754 